Draft version OCtOBER 18, 2018

Preprint typeset using $\mathrm{LTE}_{\mathrm{E}} \mathrm{X}$ style emulateapj v. 7/15/03

\title{
RELATIVISTIC STARS WITH POLOIDAL AND TOROIDAL MAGNETIC FIELDS AND MERIDIONAL FLOW
}

\author{
Kunihito IOKA ${ }^{1}$ AND Misao SASAKI ${ }^{2}$ \\ Draft version October 18, 2018
}

\begin{abstract}
We investigate stationary axisymmetric configurations of magnetized stars in the framework of general relativistic ideal magnetohydrodynamics. Our relativistic stellar model incorporates a toroidal magnetic field and meridional flow in addition to a poloidal magnetic field for the first time. The magnetic field and meridional flow are treated as perturbations, but no other approximation is made. We find that the stellar shape can be prolate rather than oblate when a toroidal field exists. We also find that, for fixed baryonic mass and total magnetic helicity, more spherical the star is, lower the energy it has. Further, we find two new types of the frame dragging effect which differ from the standard one in a rotating star or Kerr geometry. They may violate the reflection symmetry about the equatorial plane.
\end{abstract}

Subject headings: gravitation — MHD — relativity — stars: interiors — stars: magnetic fields — stars: neutron

\section{INTRODUCTION}

Equilibrium configurations of stars are fundamental to study their dynamics, such as the precession, oscillation and gravitational wave emission (Ioka 2001; Bonazzola \& Gourgoulhon 1996; Cutler 2002; Ioka \& Taniguchi 2000). Among the main factors that affect the stellar structure, the magnetic stress may be very important especially for neutron stars (Bocquet et al. 1995; Bonazzola \& Gourgoulhon 1996; Ioka 2001; Konno, Obata, \& Kojima 1999). While most neutron stars have magnetic fields of $\sim 10^{12}-10^{13} \mathrm{G}$, there is growing evidence for the existence of super-magnetized neutron stars with $\sim 10^{14}-10^{15} \mathrm{G}$, the so-called magnetars, and their birthrate is estimated to be high, $\gtrsim 10 \%$ of all neutron stars (Kouveliotou et al. 1998; Duncan \& Thompson 1992; Mereghetti et al. 2002; Thompson 2001). Larger magnetic fields $\gtrsim 10^{16} \mathrm{G}$ may be generated by the helical dynamo inside a new born neutron star (Duncan \& Thompson 1992; Thompson \& Duncan 1993). Even the maximum field strength allowed by the virial theorem $\sim 10^{18} \mathrm{G}$ could be achieved if the central engine of gamma-ray bursts are magnetars (Usov 1992; Nakamura 1998; Kluźniak \& Ruderman 1998; Wheeler, Höflich, \& Wang 2000). In a previous paper (Ioka \& Sasaki 2003), we presented a formalism to study the equilibrium configurations of magnetars. In this paper, based on this formalism, we calculate the actual configurations of magnetars.

There has been some work on stationary axisymmetric configurations of magnetized stars in the framework of general relativistic magnetohydrodynamics (MHD), but allowing the existence of only a poloidal magnetic field (Bonazzola et al. 1993; Bocquet et al. 1995; Konno, Obata, \& Kojima 1999; Cardall, Prakash, \& Lattimer 2001). The reason is that the existence of only a poloidal field is compatible with the circularity of the spacetime (Gourgoulhon \& Bonazzola 1993; Oron 2002), and once the circularity is assumed, the spacetime metric becomes substantially simple. In a circular spacetime, there exists a family of two-surfaces everywhere orthogonal to the plane defined by the two Killing vectors associated with stationarity $\eta^{\mu}=(\partial / \partial t)^{\mu}$ and axisymmetry $\xi^{\mu}=(\partial / \partial \varphi)^{\mu}$ (Papapetrou 1966; Carter 1969, 1973). Thus one may choose the coordinates $\left(x^{\mu}\right)=\left(t, x^{1}, x^{2}, \varphi\right)$ such that the metric components $g_{01}, g_{02}, g_{31}$ and $g_{32}$ are identically zero. As a consequence, the problem is simplified dramatically. However non-negligible toroidal magnetic fields are likely to exist in nature. In addition, a meridional flow may also exist in the interior of a neutron star, which also violates the circularity of the spacetime (Gourgoulhon \& Bonazzola 1993; Oron 2002). Thus, we have to consider noncircular spacetimes.

The problem to obtain an equilibrium configuration of a magnetized star can be separated into two parts. The first part is the matter and electromagnetic field equations in a given spacetime geometry (e.g., Zanotti \& Rezzolla 2002; Rezzolla, Ahmedov, \& Miller 2001a, b). The second part is the Einstein equations which determine the spacetime geometry under a given configuration of matter and electromagnetic fields. In Ioka \& Sasaki (2003), we formulated the first problem, i.e., the equations of motion for the matter and electromagnetic fields in a curved spacetime. We reduced basic equations to a single differential equation, the so-called Grad-Shafranov (GS) equation for the magnetic flux function in a noncircular (i.e., the most general) stationary axisymmetric spacetime.

In this paper, we develop a relativistic stellar model in which both a toroidal magnetic field and meridional flow are incorporated, in addition to a poloidal magnetic field, in a self-consistent way for the first time. We solve the GS equation and the perturbed Einstein equations simultaneously to determine the effects of toroidal fields and meridional flows on the spacetime geometry and stellar structure. We assume that magnetic fields are weak compared with gravity.

\footnotetext{
${ }^{1}$ Department of Earth and Space Science, Osaka University, Toyonaka 560-0043, Japan

2 Yukawa Institute for Theoretical Physics, Kyoto University, Kyoto 606-8502, Japan Electronic address: ioka@vega.ess.sci.osaka-u.ac.jp, misao@yukawa.kyoto-u.ac.jp
} 
This assumption is valid as long as the magnetic field strength is smaller than the maximum value allowed by the virial theorem $\sim 10^{18} \mathrm{G}$ (Bocquet et al. 1995; Bonazzola \& Gourgoulhon 1996). We therefore treat the magnetic field as a small perturbation on an already-known non-magnetized, non-rotating configuration. Our approach is similar to that developed for slowly rotating stars (Chandrasekhar 1933; Hartle 1967; Hartle \& Thorne 1968; Chandrasekhar \& Miller 1974), in which the perturbation parameter is the angular velocity, whereas it is the magnetic flux function in our case.

This paper is organized as follows. In $\S 2$ we briefly review general relativistic ideal MHD in stationary axisymmetric spacetimes and recapitulate the GS equation obtained in Ioka \& Sasaki (2003). In $\S$ [3 we take a weak magnetic field limit of the GS equation. This makes it possible to solve the GS equation by separation of variables. In $\S$ 政e derive the perturbed Einstein equations for the metric perturbations. In $\S$ [ we numerically solve the GS equation and the perturbed Einstein equations to obtain the magnetic field and fluid flow structure, the mass shift due to the magnetic field, the deformation of stars, and the frame dragging effects. Finally, we summarize our results in $\S[$ [

We use the units $c=G=k_{B}=1$. Greek indices $(\mu, \nu, \alpha, \beta, \cdots)$ run from 0 to 3 , small Latin indices $(i, j, k, \cdots)$ from 1 to 3 , and capital Latin indices $(A, B, C, \cdots)$ from 1 to 2 , where $x^{0}=t$ and $x^{3}=\varphi$. The signature of the 4 -metric is $(-,+,+,+)$.

\section{GENERAL RELATIVISTIC IDEAL MHD IN STATIONARY AXISYMMETRIC SPACETIMES}

The ideal MHD equations for a stationary axisymmetric system can be reduced to a single equation, the relativistic Grad-Shafranov (GS) equation (Ioka \& Sasaki 2003; Punsly 2001). It is a second-order, nonlinear partial differential equation for a quantity called the flux function $\Psi$. The flux function $\Psi$ is such that it is constant over each surface generated by rotating the magnetic field lines (or equivalently the flow lines) about the axis of symmetry and the GS equation determines the transfield equilibrium. Any physical quantities can be calculated from the solution $\Psi$ of the GS equation. The relativistic GS equation in a noncircular (i.e., the most general) stationary axisymmetric spacetime was derived in Ioka \& Sasaki (2003). In this section, we briefly review the derivation. The weak magnetic field limit of the GS equation is given in $\S[$

\subsection{General relativistic MHD equations}

The basic equations for general relativistic ideal MHD are as follows (Lichnerowicz 1967; Novikov \& Thorne 1973; Bekenstein \& Oron 1978). Baryons are conserved,

$$
\left(\rho u^{\mu}\right)_{; \mu}=0
$$

where $\rho$ is the rest mass density (i.e., the baryon mass times the baryon number density) and $u^{\mu}$ is the fluid 4-velocity with $u_{\mu} u^{\mu}=-1$. The electromagnetic field is governed by the Maxwell equations,

$$
\begin{aligned}
F_{[\mu \nu ; \alpha]} & =0, \\
F_{; \nu}^{\mu \nu} & =4 \pi J^{\mu},
\end{aligned}
$$

where $F_{\mu \nu}$ and $J^{\mu}$ are the field strength tensor and the electric current 4-vector, respectively. Equation (2) implies the existence of a vector potential $A_{\mu} ; F_{\mu \nu}=A_{\nu, \mu}-A_{\mu, \nu}$. The electric and magnetic fields in the fluid rest frame are defined as

$$
\begin{aligned}
& E_{\mu}=F_{\mu \nu} u^{\nu}, \\
& B_{\mu}=-\frac{1}{2} \epsilon_{\mu \nu \alpha \beta} u^{\nu} F^{\alpha \beta},
\end{aligned}
$$

where $\epsilon_{\mu \nu \alpha \beta}$ is the Levi-Civita antisymmetric unit tensor with $\epsilon_{0123}=\sqrt{-g}$. In the ideal MHD, we assume the perfect conductivity, so that

$$
E_{\mu}=F_{\mu \nu} u^{\nu}=0 .
$$

The equations of motion for the fluid are given by $T^{\mu \nu}{ }_{; \nu}=0$, where $T^{\mu \nu}$ is the total energy momentum tensor of the fluid and the electromagnetic field,

$$
T^{\mu \nu}=(\rho+\rho \epsilon+p) u^{\mu} u^{\nu}+p g^{\mu \nu}+\frac{1}{4 \pi}\left[\left(u^{\mu} u^{\nu}+\frac{1}{2} g^{\mu \nu}\right) B^{2}-B^{\mu} B^{\nu}\right] .
$$

Here $\epsilon$ and $p$ are the internal energy per unit mass and pressure, respectively, $B^{2}:=B^{\mu} B_{\nu}$ and we have used the perfect conductivity condition (6). Assuming local thermodynamic equilibrium, the first law of thermodynamics is given by

$$
d \epsilon=-p d\left(\frac{1}{\rho}\right)+T d S,
$$

where $S$ and $T$ are the entropy per unit mass and the temperature. Finally we supply the equation of state,

$$
p=p(\rho, S) .
$$




\subsection{Conservation laws in the axisymmetric stationary case}

We take $\eta^{\mu}=(\partial / \partial t)^{\mu}$ and $\xi^{\mu}=(\partial / \partial \varphi)^{\mu}$ so that $x^{0}=t$ and $x^{3}=\varphi$ are the time and azimuthal coordinates associated with the Killing vectors $\eta^{\mu}$ and $\xi^{\mu}$, respectively. Thus all physical quantities are independent of $t$ and $\varphi$.

In a stationary axisymmetric MHD system, there exist five conserved quantities along each flow line constructed from the energy-momentum tensor (Bekenstein \& Oron 1978, 1979; Ioka \& Sasaki 2003): $D, L, \Omega, C$ and $S$. By exploiting the gauge freedom to make $A_{\mu, \nu} \eta^{\nu}=A_{\mu, 0}=0$ and $A_{\mu, \nu} \xi^{\nu}=A_{\mu, 3}=0$, we can show that the magnetic potential $\Psi:=A_{\mu} \xi^{\mu}=A_{3}$ as well as the electric potential $\Phi:=A_{\mu} \eta^{\mu}=A_{0}$ are constant along each flow line, i.e., $u^{\mu} \Psi_{, \mu}=u^{\mu} \Phi_{, \mu}=0$. Henceforth we label the flow line by $\Psi$, which we will refer to as the flux function. The $\Psi=$ const. surfaces are called the flux surfaces, which are generated by rotating the magnetic field lines (or the flow lines) about the axis of symmetry. Then one can show that

$$
\begin{aligned}
F_{03} & =0, \\
F_{0 A} & =\Omega F_{A 3}, \\
F_{31} & =-\Psi_{, 1}=C \sqrt{-g} \rho u^{2}, \\
F_{23} & =\Psi_{, 2}=C \sqrt{-g} \rho u^{1}, \\
F_{12} & =C \sqrt{-g} \rho\left(u^{3}-\Omega u^{0}\right),
\end{aligned}
$$

where $\Omega(\Psi)$ and $C(\Psi)$ are conserved along each flow line and hence are functions of the flux function $\Psi$. It may be useful to rewrite the above equations as

$$
B^{\mu}=-C \rho\left[\left(u_{0}+\Omega u_{3}\right) u^{\mu}+\eta^{\mu}+\Omega \xi^{\mu}\right] .
$$

In addition we can show that $E(\Psi), L(\Psi)$ and $D(\Psi)$ are also conserved along each flow line where

$$
\begin{aligned}
-D & =\mu\left(u_{0}+\Omega u_{3}\right), \\
-E & =\left(\mu+\frac{B^{2}}{4 \pi \rho}\right) u_{0}+C\left(u_{0}+\Omega u_{3}\right) \frac{B_{0}}{4 \pi}, \\
L & =\left(\mu+\frac{B^{2}}{4 \pi \rho}\right) u_{3}+C\left(u_{0}+\Omega u_{3}\right) \frac{B_{3}}{4 \pi},
\end{aligned}
$$

and

$$
\mu=1+\epsilon+\frac{p}{\rho},
$$

is the enthalpy per unit mass. These conserved quantities are not mutually independent but there is a relation among them,

$$
D=E-\Omega L .
$$

Except for the entropy per unit mass $S(\Psi)$, there are no perfectly relevant physical interpretations of these quantities. Nevertheless, by considering several limiting cases, we may associate them with terms that describe their qualitative nature. We may call $D(\Psi)$ the fluid energy per unit mass, $E(\Psi)$ the total energy per unit mass, $L(\Psi)$ the total angular momentum per unit mass, $\Omega(\Psi)$ the angular velocity, and $C(\Psi)$ the magnetic field strength relative to the magnitude of meridional flow. Since these conserved quantities are essentially the first integrals of the equations of motion, specification of these functions characterizes the configuration of the electromagnetic field and fluid flow.

$$
\text { 2.3. }(2+1)+1 \text { formalism }
$$

To describe the metric of the noncircular (the most general) stationary axisymmetric spacetime in a covariant fashion, we adopt the $(2+1)+1$ formalism developed by Gourgoulhon \& Bonazzola (1993). Note that this formalism is different from the $(2+1)+1$ formalism by Maeda, Sasaki, Nakamura, \& Miyama (1980) and by Sasaki (1984; see also Nakamura, Oohara, \& Kojima 1987), which is suitable to the axisymmetric gravitational collapse. Here we adopt the formalism by Gourgoulhon \& Bonazzola (1993) because it is more convenient for a spacetime which is not only axisymmetric but also stationary.

Let $n^{\mu}$ be the unit timelike 4 -vector orthogonal to the $t=$ const. hypersurface $\Sigma_{t}$ and oriented in the direction of increasing $t$,

$$
n_{\mu}=-N t_{, \mu}
$$

The 3-metric induced by $g_{\mu \nu}$ on $\Sigma_{t}$ is given by

$$
h_{\mu \nu}=g_{\mu \nu}+n_{\mu} n_{\nu} .
$$

Similarly, let $m_{\mu}$ be the unit spacelike 4 -vector orthogonal to the $t=$ const. and $\varphi=$ const. hypersurface $\Sigma_{t \varphi}$ and oriented in the direction of increasing $\varphi$,

$$
m_{\mu}=M h_{\mu}{ }^{\nu} \varphi_{, \nu}=M \varphi_{\mid \mu}
$$


where the vertical stroke $\mid$ denotes the covariant derivative associated with the 3 -metric $h_{\mu \nu}$. The induced 2 -metric on $\Sigma_{t \varphi}$ is given by

$$
H_{\mu \nu}=h_{\mu \nu}-m_{\mu} m_{\nu}=g_{\mu \nu}+n_{\mu} n_{\nu}-m_{\mu} m_{\nu}
$$

The covariant derivative associated with the 2 -metric $H_{\mu \nu}$ is denoted by a double vertical stroke $\|$. There is a relation between the determinants as $\sqrt{-g}=N \sqrt{h}=N M \sqrt{H}$.

Any 4-vector can be decomposed into its projection onto $\Sigma_{t \varphi}$, the component parallel to $n_{\mu}$ and that to $m_{\mu}$. The Killing vectors are decomposed as

$$
\begin{aligned}
& \eta^{\mu}=N n^{\mu}-N^{\mu}=N n^{\mu}-M N^{\varphi} m^{\mu}-N_{\Sigma}{ }^{\mu}, \\
& \xi^{\mu}=M m^{\mu}-M_{\Sigma}{ }^{\mu},
\end{aligned}
$$

where the shift vector $N^{\mu}$ is (minus) the projection of $\eta^{\mu}$ onto $\Sigma_{t}, M_{\Sigma}{ }^{\mu}$ is (minus) the projection of $\xi^{\mu}$ onto $\Sigma_{t \varphi}$, and $N_{\Sigma}{ }^{\mu}$ is the projection of $N^{\mu}$ onto $\Sigma_{t \varphi}$. For our choice of the coordinates, i.e., for $x^{0}=t$ and $x^{3}=\varphi$, the component expressions for $n^{\mu}$ and $m^{\mu}$ are

$$
\begin{aligned}
n^{\mu} & =\left(\frac{1}{N}, \frac{N^{1}}{N}, \frac{N^{2}}{N}, \frac{N^{\varphi}}{N}\right), \\
m^{\mu} & =\left(0, \frac{M_{\Sigma}^{1}}{M}, \frac{M_{\Sigma}^{2}}{M}, \frac{1}{M}\right) .
\end{aligned}
$$

Note that $N_{\Sigma}^{\mu}=\left(0, N_{\Sigma}^{1}, N_{\Sigma}^{2}, 0\right)$ and $N^{A}=N_{\Sigma}^{A}+N^{\varphi} M_{\Sigma}{ }^{A}$. We can express the 4-metric $g_{\mu \nu}$ in terms of $N, N^{\varphi}$, $N_{\Sigma}{ }^{A}, M, M_{\Sigma}{ }^{A}$ and $H_{A B}$ as

$$
\begin{aligned}
g_{\mu \nu} d x^{\mu} d x^{\nu} & =-\left[N^{2}-M^{2}\left(N^{\varphi}\right)^{2}-N_{\Sigma A} N_{\Sigma}{ }^{A}\right] d t^{2}-2\left(M^{2} N^{\varphi}-N_{\Sigma}{ }^{A} M_{\Sigma A}\right) d t d \varphi \\
& -2 N_{\Sigma A} d t d x^{A}+H_{A B} d x^{A} d x^{B}-2 M_{\Sigma A} d \varphi d x^{A}+\left(M^{2}+M_{\Sigma A} M_{\Sigma}{ }^{A}\right) d \varphi^{2},
\end{aligned}
$$

where the functions $N, N^{\varphi}, N_{\Sigma}{ }^{A}, M, M_{\Sigma}{ }^{A}$ and $H_{A B}$ depend only on the coordinate $\left(x^{1}, x^{2}\right)$. We note that the presence of $N_{\Sigma}^{\mu}$ and $M_{\Sigma}^{\mu}$ characterizes the degree of noncircularity of the spacetime. Since we only assume that physical quantities are independent of $x^{0}=t$ and $x^{3}=\varphi$, the metric $g_{\mu \nu}$ in equation (29) has some degrees of freedom in the choice of coordinates. Nevertheless, the norms $N_{\Sigma}{ }^{\mu} N_{\Sigma \mu}$ and $M_{\Sigma}{ }^{\mu} M_{\Sigma \mu}$ cannot be set equal to zero for a general noncircular spacetime. In $\S 2.5$ the GS equation will be given as an equation projected onto $\Sigma_{t \varphi}$.

\subsection{Physical quantities from flux function $\Psi$}

Provided that the metric $g_{\mu \nu}$ is given and the conserved quantities $E(\Psi)$ (or $\left.D(\Psi)\right), L(\Psi), \Omega(\Psi), C(\Psi)$ and $S(\Psi)$ are given as functions of $\Psi$, all the physical quantities can be evaluated once the (effectively 2-dimensional) configuration of the flux function $\Psi$ is known (Ioka \& Sasaki 2003).

The fluid 4-velocity is expressed as

$$
u^{\mu}=u_{\eta}\left(\eta^{\mu}+\Omega \xi^{\mu}\right)+u_{\xi}\left(\xi^{\mu}+\Theta \eta^{\mu}\right)+\tilde{u}_{\Sigma}{ }^{\mu},
$$

where

$$
\begin{aligned}
u_{\eta} & =\frac{E-\Omega L}{G_{\eta} \mu}-\frac{\tilde{N}_{\Sigma}}{G_{\eta}}=\frac{D}{G_{\eta} \mu}-\frac{\tilde{N}_{\Sigma}}{G_{\eta}} \\
u_{\xi} & =-\frac{(L-\Theta E)}{G_{\xi} \mu}\left(\frac{4 \pi \mu}{G_{\eta} C^{2} \rho}\right)\left(1-\frac{4 \pi \mu}{G_{\eta} C^{2} \rho}\right)^{-1}+\frac{\tilde{M}_{\Sigma}}{G_{\xi}}, \\
\tilde{u}_{\Sigma}{ }^{\mu} & =\frac{1}{N M C \rho} \epsilon^{\mu \nu} \Psi_{, \nu}
\end{aligned}
$$

and we define

$$
\begin{aligned}
\Theta & =-\frac{\xi_{\nu}\left(\eta^{\nu}+\Omega \xi^{\nu}\right)}{\eta_{\mu}\left(\eta^{\mu}+\Omega \xi^{\mu}\right)}=-\frac{g_{03}+\Omega g_{33}}{g_{00}+\Omega g_{03}}, \\
G_{\eta} & =-\left(\eta_{\mu}+\Omega \xi_{\mu}\right)\left(\eta^{\mu}+\Omega \xi^{\mu}\right)=-\left(g_{00}+2 \Omega g_{03}+\Omega^{2} g_{33}\right), \\
G_{\xi} & =\left(\xi_{\mu}+\Theta \eta_{\mu}\right)\left(\xi^{\mu}+\Theta \eta^{\mu}\right)=g_{33}+2 \Theta g_{03}+\Theta^{2} g_{00}, \\
\tilde{N}_{\Sigma} & =\tilde{u}_{\Sigma}^{\mu}\left(N_{\Sigma \mu}+\Omega M_{\Sigma \mu}\right), \\
\tilde{M}_{\Sigma} & =\tilde{u}_{\Sigma}^{\mu}\left(M_{\Sigma \mu}+\Theta N_{\Sigma \mu}\right), \\
\epsilon^{\mu \nu} & =\epsilon^{\mu \nu \alpha \beta} n_{\alpha} m_{\beta} .
\end{aligned}
$$

In equation (30), the orthogonality $\left(\eta^{\mu}+\Omega \xi^{\mu}\right)\left(\xi_{\mu}+\Theta \eta_{\mu}\right)=0$ is satisfied. Note that $M_{\mathrm{Alf}}^{2}:=4 \pi \mu / G_{\eta} C^{2} \rho$ is the square of the effective Alfvén Mach number $M_{\text {Alf }}$. At the Alfvén point $M_{\text {Alf }}=1$, the numerator $L-\Theta E$ in equation (32) should vanish to keep the velocity $u_{\xi}$ finite. 
Thus, from equations (30) - (33), given the metric $g_{\mu \nu}$ and the conserved functions $E(\Psi)($ or $D(\Psi)), L(\Psi), \Omega(\Psi)$, $C(\Psi)$ and $S(\Psi)$, if the density $\rho$ and the enthalpy $\mu$ are additionally known (see below), the fluid 4 -velocity $u^{\mu}$ can be obtained from the flux function $\Psi$ and its first derivatives $\Psi_{, A}$. The magnetic field is also calculated from the flux function $\Psi$ by using equation (15). The $(2+1)+1$ decomposition of the fluid 4 -velocity is performed with equations (25), (26) and (30) as

$$
u^{\mu}=u_{n} n^{\mu}+u_{m} m^{\mu}+u_{\Sigma}^{\mu},
$$

where

$$
\begin{aligned}
u_{n} & =N\left(u_{\eta}+\Theta u_{\xi}\right), \\
u_{m} & =M\left[\left(\Omega-N^{\varphi}\right) u_{\eta}+\left(1-N^{\varphi} \Theta\right) u_{\xi}\right], \\
u_{\Sigma}{ }^{\mu} & =\tilde{u}_{\Sigma}{ }^{\mu}-\left(u_{\eta}+\Theta u_{\xi}\right) N_{\Sigma}{ }^{\mu}-\left(u_{\xi}+\Omega u_{\eta}\right) M_{\Sigma}{ }^{\mu} .
\end{aligned}
$$

The pressure $p$, the internal energy $\epsilon$, the enthalpy $\mu$ and the temperature $T$ are functions of the density $\rho$ and the entropy $S$ from equations (19), (8) and (9). Hence, given $S$ as a function of $\Psi$, the only remaining quantity to be known is the density $\rho$. The density $\rho$ is determined by the normalization of the 4-velocity, which yields

$$
-\frac{D^{2}}{G_{\eta} \mu^{2}}+\frac{(4 \pi)^{2}(L-\Theta E)^{2}}{G_{\xi} G_{\eta}{ }^{2} C^{4} \rho^{2}}\left(1-\frac{4 \pi \mu}{G_{\eta} C^{2} \rho}\right)^{-2}+\frac{H^{A B} \Psi_{, A} \Psi_{, B}}{N^{2} M^{2} C^{2} \rho^{2}}+\frac{\tilde{N}_{\Sigma}^{2}}{G_{\eta}}-\frac{\tilde{M}_{\Sigma}^{2}}{G_{\xi}}=-1 .
$$

This equation is called the wind equation.

The following components of the electric current are also calculated from the flux function,

$$
\begin{aligned}
& J^{0}=\frac{1}{4 \pi N M \sqrt{H}}\left(N M \sqrt{H} F^{0 A}\right)_{, A}=\frac{1}{4 \pi N M}\left(N M F^{0 A}\right)_{\| A}, \\
& J^{3}=\frac{1}{4 \pi N M \sqrt{H}}\left(N M \sqrt{H} F^{3 A}\right)_{, A}=\frac{1}{4 \pi N M}\left(N M F^{3 A}\right)_{\| A},
\end{aligned}
$$

where

$$
\begin{aligned}
& F^{0 A}=\left(g^{00} g^{A B}-g^{0 B} g^{A 0}\right) \Omega \Psi_{, B}+\left(g^{0 B} g^{A 3}-g^{03} g^{A B}\right) \Psi_{, B}+\left(g^{01} g^{A 2}-g^{02} g^{A 1}\right) F_{12} \\
& =-\frac{1}{N^{2}}\left[H^{A B}+\frac{M_{\Sigma}^{A} M_{\Sigma}^{B}}{M^{2}}\right] \Omega \Psi_{, B}+\frac{1}{N^{2}}\left[N^{\varphi} H^{A B}-\frac{M_{\Sigma}^{A} N_{\Sigma}^{B}}{M^{2}}\right] \Psi_{, B} \\
& -\left[\left(\frac{N_{\Sigma}^{B}+N^{\varphi} M_{\Sigma}^{B}}{N^{2}}\right) \epsilon_{B}{ }^{A}+\frac{M_{\Sigma}^{A} N_{\Sigma}^{B} M_{\Sigma}^{C} \epsilon_{B C}}{N^{2} M^{2}}\right] \frac{F_{12}}{\sqrt{H}}, \\
& F^{3 A}=\left(g^{30} g^{A B}-g^{3 B} g^{A 0}\right) \Omega \Psi_{, B}+\left(g^{3 B} g^{A 3}-g^{33} g^{A B}\right) \Psi_{, B}+\left(g^{31} g^{A 2}-g^{32} g^{A 1}\right) F_{12} \\
& =-\frac{1}{N^{2}}\left[N^{\varphi} H^{A B}-\frac{N_{\Sigma}^{A} M_{\Sigma}^{B}}{M^{2}}\right] \Omega \Psi_{, B}-\left[\left(\frac{1}{M^{2}}-\left(\frac{N^{\varphi}}{N}\right)^{2}\right) H^{A B}-\frac{N_{\Sigma}{ }^{A} N_{\Sigma}^{B}}{N^{2} M^{2}}\right] \Psi_{, B} \\
& +\left[\left\{\left(\frac{1}{M^{2}}-\left(\frac{N^{\varphi}}{N}\right)^{2}\right) M_{\Sigma}^{B}-\frac{N^{\varphi} N_{\Sigma}^{B}}{N^{2}}\right\} \epsilon_{B}{ }^{A}+\frac{N_{\Sigma}^{A} N_{\Sigma}^{B} M_{\Sigma}^{C} \epsilon_{B C}}{N^{2} M^{2}}\right] \frac{F_{12}}{\sqrt{H}}, \\
& F_{12}=C \sqrt{-g} \rho\left(u^{3}-\Omega u^{0}\right)=C \sqrt{-g} \rho u_{\xi}(1-\Omega \Theta) .
\end{aligned}
$$

Thus, $J^{0}$ and $J^{3}$ are expressed in terms of $\Psi$ and its first and second derivatives.

\subsection{Relativistic Grad-Shafranov equation}

The equation for the flux function $\Psi$, that is, the GS equation is given by (Ioka \& Sasaki 2003)

$$
J^{3}-\Omega J^{0}+\frac{1}{N M C} \epsilon^{A B}\left(\mu u_{\Sigma} A\right)_{\| B}-\rho\left(u_{\eta}+\Theta u_{\xi}\right)\left[\frac{d E}{d \Psi}-\Lambda \frac{d(C \Omega)}{d \Psi}\right]+\rho\left(u_{\xi}+\Omega u_{\eta}\right)\left[\frac{d L}{d \Psi}-\Lambda \frac{d C}{d \Psi}\right]+\rho T \frac{d S}{d \Psi}=0,(5
$$

where the double vertical stroke $\|$ denotes covariant differentiation with respect to the 2 -metric $H_{A B}$, and we have defined the auxiliary quantity

$$
\Lambda=\frac{1}{4 \pi}\left(u_{0} B_{3}-u_{3} B_{0}\right)=\frac{1}{4 \pi} C \rho\left(G_{\xi} u_{\xi}-\tilde{M}_{\Sigma}\right)\left(g_{00}+\Omega g_{03}\right) .
$$

In the previous subsection, we have seen that $u_{\eta}, u_{\xi}, u_{\Sigma}{ }^{A}, \Theta, \rho, \mu, T, J^{0}$ and $J^{3}$ are all expressed in terms of $\Psi$ and its derivatives, given the conserved functions $E(\Psi)$ (or $D(\Psi)$ ), $L(\Psi), \Omega(\Psi), C(\Psi)$ and $S(\Psi)$, and the metric $g_{\mu \nu}$. Thus, in order to obtain the configuration of matter and electromagnetic fields in a curved spacetime, we have only to solve the GS equation (50) with the aid of the wind equation (44). 


\section{GRAD-SHAFRANOV EQUATION IN THE WEAK MAGNETIC FIELD LIMIT}

It is formidable to solve the GS equation (50) in a general case because of its high non-linearity. However, it becomes tractable if we take the weak magnetic field limit. In this section, we derive the GS equation in the weak field limit with the flux function $\Psi$ being the perturbation parameter. This perturbation method is essentially similar to that developed by Chandrasekhar (1933; Chandrasekhar \& Miller 1974) and Hartle (1967; Hartle \& Thorne 1968) for slowly rotating stars, in which the perturbation parameter is the angular velocity.

\subsection{Zeroth order}

In Ioka \& Sasaki (2003), we showed that no magnetic field limit is obtained by letting $\Psi \rightarrow 0$ and $C \rightarrow \infty$. In this limit, the GS equation (50) and the wind equation (44) are reduced to the integrability condition and the Bernouilli's equation for the rotating isentropic $(d S / d \Psi=0)$ star, respectively (Ioka \& Sasaki 2003). Thus a rotating fluid star is the zeroth order configuration in the weak magnetic field approximation.

Let us further suppose that there is no rotation $\Omega=0$ at the zeroth order in $\Psi$. Then the background metric $\bar{g}_{\mu \nu}$ is spherically symmetric,

$$
\bar{g}_{\mu \nu} d x^{\mu} d x^{\nu}=-e^{2 \nu} d t^{2}+e^{2 \lambda} d r^{2}+r^{2}\left(d \theta^{2}+\sin ^{2} \theta d \varphi^{2}\right)
$$

and the zeroth order configuration is obtained by the Tolman-Oppenheimer-Volkoff equations,

$$
\begin{aligned}
m^{\prime} & =4 \pi r^{2} \rho(1+\epsilon), \\
p^{\prime} & =-\frac{1}{r^{2}} e^{2 \lambda} \rho \mu\left(m+4 \pi p r^{3}\right), \\
\nu^{\prime} & =-\frac{1}{\rho \mu} p^{\prime},
\end{aligned}
$$

where the prime denotes differentiation with respect to $r$, and the mass $m(r)$ inside radius $r$ is defined by

$$
e^{2 \lambda}=\left(1-\frac{2 m}{r}\right)^{-1}
$$

The value $r=R_{*}$ at which $p=0$ is the radius of the star, and the total mass of the star is given by $m\left(R_{*}\right)=M_{*}$. The boundary conditions are $m(r=0)=0$ and $2 \nu\left(r=R_{*}\right)=\ln \left(1-2 M_{*} / R_{*}\right)$.

\subsection{First order}

We perform linearization with respect to the flux function $\Psi$. To proceed, we have to specify the functional forms of the conserved functions $D(\Psi)$ (or $E(\Psi)$ ), $L(\Psi), \Omega(\Psi), C(\Psi)$ and $S(\Psi)$, which characterize the configuration of the fluid flow and the electromagnetic field. In this paper, we assume the following.

- $C(\Psi)$ : We consider the case in which the magnetic field is confined in the interior of a star. Hence, $\Psi$ must vanish at the stellar surface. Then, the Alfvén point $M_{\text {Alf }}^{2}=4 \pi \mu / G_{\eta} C^{2} \rho=1$ exists near the stellar surface $\rho \sim 0$ in equation (32) unless $C$ diverges at the stellar surface. Therefore, we assume the form

$$
C=\frac{\tilde{C}}{\Psi},
$$

where $\tilde{C}=$ const. $=O(1)$. We note that as long as the magnitude of $\tilde{C}$ is much greater than $\Psi$, the discussion below is valid, but we assume it to be $O(1)$ for the sake of order counting.

- $\Omega(\Psi)$ : Since our primary interest is the effect of magnetic fields on the stellar structure, we consider a slow (rigid) rotation which is of the same order as the one induced by the presence of a magnetic field. Hence we set

$$
\Omega=\text { const. }=O\left(\Psi^{2}\right) \text {. }
$$

Here, although we may consider the case $\Omega=O(\Psi)$, we choose not to do so, and assume that the rotation is subdominant. Note that the observed magnetars have long rotation periods $(\sim \sec )$, so that this assumption is justified.

- $S(\Psi)$ : We consider an isentropic star for simplicity,

$$
S=\text { const.. }
$$

- $D(\Psi)$ and $L(\Psi)$ : These functions are chosen in such a way that the GS equation becomes separable with respect to $r$ and $\theta$. Namely,

$$
\begin{aligned}
& D=D_{0}+D_{1} \Psi+D_{2}, \\
& L=\text { const. }=O(\tilde{C}),
\end{aligned}
$$

where $D_{0}=$ const. $=O(1), D_{1}=$ const. $=O(\Psi)$ and $D_{2}=$ const. $=O\left(\Psi^{2}\right)$. Note that the conserved function $L(\Psi)$ does not necessarily vanish at zeroth order at which there is no rotation. This is because $L(\Psi)$ does not exactly coincide with the angular momentum. 
With these assumptions for the conserved quantities, we can estimate the orders of the variables from equations (31) - (39), (43) and (15) as

$$
\Theta=O\left(\Psi^{2}\right), \quad u_{\eta}=O(1), \quad u_{\xi}=O\left(\Psi^{2}\right), \quad \tilde{u}_{\Sigma}^{\mu} \sim u_{\Sigma}^{\mu}=O\left(\Psi^{2}\right), \quad B_{0}=O\left(\Psi^{3}\right), \quad B_{i}=O(\Psi),
$$

where we have used the fact that the metric perturbations are $O\left(\Psi^{2}\right)$, i.e., in equation (29) we have

$$
N^{\varphi}=O\left(\Psi^{2}\right), \quad N_{\Sigma}^{\mu}=O\left(\Psi^{2}\right), \quad M_{\Sigma}^{\mu}=O\left(\Psi^{2}\right),
$$

and $g_{03}=O\left(\Psi^{2}\right)$, which will be confirmed in $\S$. Note that $N=e^{\nu}, M=r \sin \theta, H_{r r}=e^{2 \lambda}$ and $H_{\theta \theta}=r^{2}$ at zeroth order from equations (29) and (52).

Now let us derive the GS equation in the weak magnetic field limit. To $O(\Psi)$, equation (50) is reduced to

$$
J^{3}-\rho u_{\eta} \frac{d E}{d \Psi}-\rho u_{\xi} \Lambda \frac{d C}{d \Psi}=0,
$$

where, from equations (46) and (48), the electric current is given by

$$
J^{3}=\frac{1}{4 \pi \sqrt{-g}}\left(\sqrt{-g} F^{3 A}\right)_{, A}=-\frac{1}{4 \pi \sqrt{-\bar{g}}}\left(\sqrt{-\bar{g}} \frac{1}{M^{2}} H^{A B} \Psi_{, B}\right)_{, A} .
$$

Up to $O\left(\Psi^{2}\right)$, equations (35), (36), (31) and (32) are reduced, respectively, to

$$
\begin{aligned}
G_{\eta} & =-g_{00}, \\
G_{\xi} & =g_{33}, \\
u_{\eta} & =\frac{D}{\left(-g_{00}\right) \mu}, \\
u_{\xi} & =\frac{4 \pi L}{g_{00} g_{33} C^{2} \rho} .
\end{aligned}
$$

Hence the wind equation (44) is reduced to

$$
\frac{D^{2}}{\left(-g_{00}\right) \mu^{2}}=\frac{D}{\mu} u_{\eta}=\left(-g_{00}\right) u_{\eta}^{2}=1,
$$

where we note that $g_{00}$ and $g_{33}$ include perturbations up to $O\left(\Psi^{2}\right)$. Then, at $O(\Psi)$, the second term in equation (64) is given by

$$
-\rho u_{\eta} \frac{d E}{d \Psi}=-\rho u_{\eta} \frac{d(D+\Omega L)}{d \Psi}=-\rho u_{\eta} D_{1}=-\rho e^{-\nu} D_{1},
$$

where we have used $u_{\eta}=1 / \sqrt{-\bar{g}_{00}}=e^{-\nu}$ at zeroth order that follows from equation (70). Note that we have $D_{0}=\mu e^{\nu}$ at zeroth order. Similarly, from equations (51), (57) and (32), the third term in equation (64) is approximated as

$$
-\rho u_{\xi} \Lambda \frac{d C}{d \Psi}=-\frac{1}{4 \pi r^{2} \sin ^{2} \theta}\left(\frac{4 \pi L}{\tilde{C}}\right)^{2} e^{-2 \nu} \Psi .
$$

Then, by combining equations (64), (65), (71) and (72), the GS equation valid to $O(\Psi)$ is given by

$$
e^{\lambda-\nu}\left[\partial_{r}\left(e^{\nu-\lambda} \partial_{r} \Psi\right)+\frac{e^{\nu+\lambda}}{r^{2}} \sin \theta \partial_{\theta}\left(\frac{1}{\sin \theta} \partial_{\theta} \Psi\right)\right]+\left(\frac{4 \pi L}{\tilde{C}}\right)^{2} e^{2(\lambda-\nu)} \Psi+4 \pi r^{2} \sin ^{2} \theta \rho e^{2 \lambda-\nu} D_{1}=0
$$

where we have used the form of the background metric $\bar{g}_{\mu \nu}$ given in equation (52). Note that, under the assumptions for the conserved quantities we have made, the GS equation at this order is an inhomogeneous linear differential equation for $\Psi$ with the last term proportional to $D_{1}$ as a source.

Let us separate the angular variables in the GS equation. We expand the flux function $\Psi$ by the vector spherical harmonics (Regge \& Wheeler 1957; Zerilli 1970) as

$$
\Psi=\sum_{\ell=1}^{\infty} \psi_{\ell}(r) \sin \theta \frac{\partial P_{\ell}(\cos \theta)}{\partial \theta}
$$

where $P_{\ell}$ is the Legendre polynomial of degree $\ell$, and we have discarded the $\varphi$-dependence of the harmonics because of the axisymmetry. Substituting this form into equation (73), we find the equation for the dipole $\psi_{1}$ as

$$
\psi_{1}^{\prime \prime}+\left(\nu^{\prime}-\lambda^{\prime}\right) \psi_{1}^{\prime}+\left(\tilde{L}^{2} e^{-2 \nu}-\frac{2}{r^{2}}\right) e^{2 \lambda} \psi_{1}-4 \pi r^{2} \rho e^{2 \lambda-\nu} D_{1}=0,
$$

and for the higher multipoles as

$$
\psi_{\ell}^{\prime \prime}+\left(\nu^{\prime}-\lambda^{\prime}\right) \psi_{\ell}^{\prime}+\left(\tilde{L}^{2} e^{-2 \nu}-\frac{\ell(\ell+1)}{r^{2}}\right) e^{2 \lambda} \psi_{\ell}=0 \quad(\ell \geq 2),
$$


where we have introduced

$$
\tilde{L}=\frac{4 \pi L}{\tilde{C}}
$$

Equations (75) and (76) are the basic equations for the matter and electromagnetic fields for the conserved quantities of the assumed forms in the weak magnetic field limit.

Since we are interested in magnetic fields confined in the stellar interior, or interior magnetic fields with magnitude much larger than exterior magnetic fields, we assume that the magnetic field vanishes at the stellar surface. Thus, to the lowest order in $\Psi$, we require

$$
\psi_{\ell}\left(R_{*}\right)=0, \quad \psi_{\ell}^{\prime}\left(R_{*}\right)=0 \quad(\ell \geq 1) .
$$

Near the origin $r=0$, the regular solutions of equations (75) and (76) should have the behavior

$$
\psi_{\ell} \rightarrow r^{\ell+1} \alpha_{\ell} \quad(\ell \geq 1)
$$

where $\alpha_{\ell}$ is a constant.

First consider the dipole $\psi_{1}$. With the boundary conditions given above, equation (75) becomes an eigenvalue equation with $\tilde{L}$ being the eigenvalue to be determined. There will be a discrete set of eigenvalues. For a given eigenvalue, the constant $D_{1}$ then just determines the normalization of $\psi_{1}$. Now we consider the higher multipoles $\psi_{\ell}(\ell \geq 2)$. Once $\tilde{L}$ is determined, it is in general impossible to find non-trivial solutions for $\ell \geq 2$ that also satisfy the same boundary conditions (78) and (79). Thus, under our assumptions that the conserved quantities are of the forms (57) - 61) and that magnetic fields are confined in the stellar interior, the only non-trivial solutions are of dipole type. Of course, once we relax the assumption of confined magnetic fields, the existence of higher multipoles will be allowed.

In the numerical analysis, it is convenient to integrate equation (75) from the stellar surface with equation (78) as initial data. By varying $\tilde{L}$, we look for a solution that behaves as regular as possible at the origin. This procedure determines the eigenvalue $\tilde{L}$. Next we integrate equation (75) from the origin with the behavior (79) and the fixed eigenvalue $\tilde{L}$. Then the constant $\alpha_{1}$ is determined by demanding that the boundary condition at the stellar surface, equation (178), is satisfied.

\section{METRIC PERTURBATION EQUATIONS}

In this section, we derive the field equations for the metric perturbation,

$$
\Delta g_{\mu \nu}=g_{\mu \nu}-\bar{g}_{\mu \nu} .
$$

By linearizing the Einstein equations about the background metric $\bar{g}_{\mu \nu}$, we have

$$
\begin{aligned}
& \left(\Delta g_{\mu \nu ; \alpha}{ }^{; \alpha}-\Delta g_{\mu \alpha ; \nu}{ }^{; \alpha}-\Delta g_{\nu \alpha ; \mu}{ }^{; \alpha}+\Delta g_{\alpha}{ }^{\alpha} ; \mu ; \nu\right) \\
& +\bar{g}_{\mu \nu}\left(\Delta g_{\alpha \beta}{ }^{; \alpha ; \beta}-\Delta g_{\alpha}{ }^{\alpha} ; \beta^{; \beta}-\Delta g_{\alpha \beta} R^{\alpha \beta}\right)+\Delta g_{\mu \nu} R=-16 \pi \Delta T_{\mu \nu},
\end{aligned}
$$

where $\Delta T_{\mu \nu}$ is the perturbation of the energy momentum tensor, and $R_{\mu \nu}$ and $R$ are the Ricci tensor and the Ricci scalar, respectively. As shown below, the metric perturbation $\Delta g_{\mu \nu}$ is $O\left(\Psi^{2}\right)$. Our method is similar to that developed by Hartle (1967; Hartle \& Thorne 1968; Chandrasekhar \& Miller 1974) for slowly rotating relativistic stars. We obtain the field equations for the interior of the star in $\S 4.1$ and solve the exterior equations to give the junction conditions at the stellar surface in $\S 4.2$

\subsection{Interior field equations}

The study of the metric perturbation in a spherical spacetime was initiated by Regge \& Wheeler (1957) and Zerilli (1970). Because of the spherical symmetry of the background, the perturbation equations are separable in the angular variables by using ten tensor harmonics. We can eliminate some components of the metric perturbation with the aid of the gauge freedom. In the gauge used by Regge \& Wheeler (1957), the metric perturbation belonging to a given $\ell$ is of the form,

$$
\Delta g_{\mu \nu}=\left(\begin{array}{cccc}
-2 e^{2 \nu} H_{\ell} & I_{\ell} & 0 & 0 \\
I_{\ell} & \frac{2}{r} e^{4 \lambda} M_{\ell} & 0 & 0 \\
0 & 0 & 2 r^{2} K_{\ell} & 0 \\
0 & 0 & 0 & 2 r^{2} \sin ^{2} \theta K_{\ell}
\end{array}\right) P_{\ell}(\cos \theta)+\left(\begin{array}{cccc}
0 & 0 & 0 & V_{\ell} \\
0 & 0 & 0 & W_{\ell} \\
0 & 0 & 0 & 0 \\
V_{\ell} & W_{\ell} & 0 & 0
\end{array}\right) \sin \theta \frac{\partial}{\partial \theta} P_{\ell}(\cos \theta),
$$

where the first term is of parity $(-1)^{\ell}$ (even parity), the second term is of parity $(-1)^{\ell+1}$ (odd parity) and we may discard the $\varphi$-dependence of the harmonics because of the axisymmetry. Since there are fewer independent tensor harmonics for $\ell=0$ and $\ell=1$, we may further simplify the metric by adopting a gauge in which $I_{0}=0, K_{0}=0$, $V_{0}=0, W_{0}=0, K_{1}=0$ and $W_{1}=0$ (Zerilli 1970). 
The energy momentum tensor perturbation $\Delta T_{\mu \nu}$ is obtained by taking the perturbation of equation (17). The components of the magnetic field to $O(\Psi)$ are given from equations (15), (30) - (33), (62), (63) and (170) as

$$
\begin{aligned}
B^{0} & =O\left(\Psi^{3}\right) \\
B^{1} & =-\frac{2}{r^{2}} e^{-\lambda} \psi_{1} \cos \theta \\
B^{2} & =\frac{1}{r^{2}} e^{-\lambda} \psi_{1}^{\prime} \sin \theta \\
B^{3} & =\frac{\tilde{L}}{r^{2}} e^{-\nu} \psi_{1} .
\end{aligned}
$$

Since the energy momentum tensor is quadratic in $B^{\mu}$, we need $\Delta T_{\mu \nu}$ to the accuracy of $O\left(\Psi^{2}\right)$. To this order, from equations (30) - (33), (62), (63) and (70), the fluid flow is given by

$$
\begin{aligned}
& u^{0}=u_{\eta}=\frac{1}{\sqrt{-g_{00}}}, \\
& u^{1}=\frac{2}{\tilde{C} \rho r^{2}} e^{-\lambda-\nu}\left(\psi_{1}\right)^{2} \sin ^{2} \theta \cos \theta, \\
& u^{2}=-\frac{1}{\tilde{C} \rho r^{2}} e^{-\lambda-\nu} \psi_{1} \psi_{1}^{\prime} \sin ^{3} \theta \\
& u^{3}=\Omega u_{\eta}+u_{\xi}=\Omega e^{-\nu}-\frac{L}{\tilde{C} \rho r^{2}} e^{-2 \nu}\left(\psi_{1}\right)^{2} \sin ^{2} \theta .
\end{aligned}
$$

Expanding the perturbation of the enthalpy in equation (19) in the form,

$$
\frac{\Delta \mu}{\mu}=\sum_{\ell=0}^{\infty}[\Delta \ln \mu(r)]_{\ell} P_{\ell}(\cos \theta),
$$

we obtain from equations (70) and (82) the equations

$$
\begin{aligned}
& (\Delta \ln \mu)_{0}=-H_{0}-\frac{2}{3} \frac{D_{1}}{D_{0}} \psi_{1}+\frac{D_{2}}{D_{0}}, \\
& (\Delta \ln \mu)_{2}=-H_{2}+\frac{2}{3} \frac{D_{1}}{D_{0}} \psi_{1},
\end{aligned}
$$

and $(\Delta \ln \mu)_{\ell}=-H_{\ell}$ for $\ell \neq 0,2$. From the first law of thermodynamics, equation (8), with equation (19), we have the relations $(\Delta p)_{\ell}=\rho(\Delta \mu)_{\ell}$ and $(\Delta \epsilon)_{\ell}=\left(p / \rho^{2}\right)(\Delta \rho)_{\ell}$, where $(\Delta p)_{\ell},(\Delta \epsilon)_{\ell}$ and $(\Delta \rho)_{\ell}$ are the $\ell$-th pole perturbations of the pressure, the internal energy and the rest mass density, respectively. For the polytropic equation of state $p=K \rho^{1+1 / n}$, we have $(\Delta p)_{\ell}=(1+1 / n)(p / \rho)(\Delta \rho)_{\ell}$.

From the Einstein equations (81) with $\Delta g_{\mu \nu}$ given by equation (82), we obtain the following independent equations which have nonzero source terms,

$$
\begin{aligned}
& M_{0}^{\prime}=4 \pi r^{2} \mu^{2} \rho \frac{d \rho}{d p}(\Delta \ln \mu)_{0}+\frac{1}{3}\left(\tilde{L}^{2} e^{-2 \nu}+\frac{2}{r^{2}}\right)\left(\psi_{1}\right)^{2}+\frac{1}{3} e^{-2 \lambda}\left(\psi_{1}^{\prime}\right)^{2}, \quad\left(G_{00}\right) \\
& H_{0}^{\prime}=-\left[(\Delta \ln \mu)_{0}\right]^{\prime}-\frac{2}{3 \mu} e^{-\nu} D_{1} \psi_{1}^{\prime} \\
&=\left(\frac{1}{r^{2}}+8 \pi p\right) e^{4 \lambda} M_{0}+4 \pi r e^{2 \lambda} \rho \mu(\Delta \ln \mu)_{0}+\frac{1}{3 r} e^{2 \lambda}\left(\tilde{L}^{2} e^{-2 \nu}-\frac{2}{r^{2}}\right)\left(\psi_{1}\right)^{2}+\frac{1}{3 r}\left(\psi_{1}^{\prime}\right)^{2}, \quad\left(G_{11}\right) \\
& I_{1}=-\frac{32 \pi}{5} \frac{\mu}{\tilde{C}} e^{\lambda}\left(\psi_{1}\right)^{2}, \quad\left(G_{01}\right) \\
& I_{3}=\frac{16 \pi}{15} \frac{\mu}{\tilde{C}} e^{\lambda}\left(\psi_{1}\right)^{2}, \quad\left(G_{01}\right) \\
& V_{1}^{\prime \prime}-\left(\nu^{\prime}+\lambda^{\prime}\right) V_{1}^{\prime}-\frac{2}{r}\left(\nu^{\prime}+\lambda^{\prime}+\frac{1}{r}\right) V_{1}=\frac{64 \pi}{5} \frac{\tilde{L}}{\tilde{C}} \mu e^{2 \lambda-\nu}\left(\psi_{1}\right)^{2}-16 \pi \rho \mu r^{2} e^{2 \lambda} \Omega, \quad\left(G_{03}\right) \\
& V_{3}^{\prime \prime}-\left(\nu^{\prime}+\lambda^{\prime}\right) V_{3}^{\prime}-\frac{2}{r}\left(\nu^{\prime}+\lambda^{\prime}+\frac{1}{r}+\frac{5}{r} e^{2 \lambda}\right) V_{3}=-\frac{32 \pi}{15} \frac{\tilde{L}}{\tilde{C}} \mu e^{2 \lambda-\nu}\left(\psi_{1}\right)^{2}, \quad\left(G_{03}\right) \\
& W_{2}=-\frac{2}{3} \tilde{L} e^{\lambda-\nu}\left(\psi_{1}\right)^{2}, \quad\left(G_{13}\right) \\
& H_{2}+\frac{1}{r} e^{2 \lambda} M_{2}=\frac{2}{3} e^{-2 \lambda}\left(\psi_{1}^{\prime}\right)^{2}-\frac{2}{3} \tilde{L}^{2} e^{-2 \nu}\left(\psi_{1}\right)^{2}, \quad\left(G_{22}-G_{33} / \sin ^{2} \theta\right)
\end{aligned}
$$




$$
\begin{gathered}
\left(\nu^{\prime}+\frac{1}{r}\right) K_{2}^{\prime}-\frac{2}{r^{2}} e^{2 \lambda} K_{2}+\frac{1}{r} H_{2}^{\prime}-\frac{3}{r^{2}} e^{2 \lambda} H_{2}-\frac{1}{r}\left(\frac{1}{r^{2}}+8 \pi p\right) e^{4 \lambda} M_{2} \\
=4 \pi e^{2 \lambda} \rho \mu(\Delta \ln \mu)_{2}-\frac{1}{3 r^{2}} e^{2 \lambda}\left(\tilde{L}^{2} e^{-2 \nu}+\frac{4}{r^{2}}\right)\left(\psi_{1}\right)^{2}-\frac{1}{3 r^{2}}\left(\psi_{1}^{\prime}\right)^{2}, \quad\left(G_{11}\right) \\
K_{2}^{\prime}+H_{2}^{\prime}-\left(\frac{1}{r}-\nu^{\prime}\right) H_{2}-\frac{1}{r}\left(\frac{1}{r}+\nu^{\prime}\right) e^{2 \lambda} M_{2}=\frac{4}{3 r^{2}} \psi_{1} \psi_{1}^{\prime}, \quad\left(G_{12}\right)
\end{gathered}
$$

where we have indicated the component of the field equation in the parentheses at the end of each equation from which it is derived. The remaining components of the Einstein equations that are not listed in the above are either trivially satisfied or not independent from the above set because of the Bianchi identities (Zerilli 1970). To avoid the cancellation of significant digits in the numerical calculation, it is convenient to define

$$
Y_{2}=H_{2}+K_{2}-\frac{1}{6} e^{-2 \lambda}\left(\psi_{1}^{\prime}\right)^{2}-\frac{2}{3 r} e^{-2 \lambda} \psi_{1} \psi_{1}^{\prime}-\frac{2}{3 r^{2}}\left(\psi_{1}\right)^{2},
$$

and solve for $Y_{2}$ instead of $K_{2}$. With equations (93) and (101), we can rewrite equations (102) and (103) as

$$
\begin{aligned}
& Y_{2}^{\prime}+2 \nu^{\prime} H_{2}=\nu^{\prime} e^{-2 \lambda}\left(\psi_{1}^{\prime}\right)^{2}+\frac{1}{3}\left[\tilde{L}^{2} e^{-2 \nu}+\frac{2}{r}\left(\nu^{\prime}+\lambda^{\prime}\right) e^{-2 \lambda}-\frac{4 m}{r^{3}}\right] \psi_{1} \psi_{1}^{\prime} \\
& \quad-\frac{2}{3} \nu^{\prime} \tilde{L}^{2} e^{-2 \nu}\left(\psi_{1}\right)^{2}-\frac{4 \pi}{3} r \rho e^{-\nu} D_{1}\left(r \psi_{1}^{\prime}+2 \psi_{1}\right), \\
& H_{2}^{\prime}+\frac{2}{r^{2} \nu^{\prime}} e^{2 \lambda} Y_{2}+\left[2 \nu^{\prime}-\frac{1}{\nu^{\prime}} e^{2 \lambda}\left(4 \pi \rho \mu-\frac{2 m}{r^{3}}\right)\right] H_{2} \\
& =\frac{2}{3} \nu^{\prime} e^{-2 \lambda}\left(\psi_{1}^{\prime}\right)^{2}+\frac{4}{3 r^{2}} \psi_{1} \psi_{1}^{\prime}+\frac{1}{3}\left(-2 \nu^{\prime}+\frac{1}{r^{2} \nu^{\prime}} e^{2 \lambda}\right) \tilde{L}^{2} e^{-2 \nu}\left(\psi_{1}\right)^{2}-\frac{8 \pi}{3 \nu^{\prime}} \rho e^{2 \lambda-\nu} D_{1} \psi_{1} .
\end{aligned}
$$

We have six differential equations (94), (95), (98), (99), (105) and (106) for six unknown functions; $M_{0},(\Delta \ln \mu)_{0}, V_{1}$, $V_{3}, Y_{2}$ and $H_{2}$. A practical method to solve these equations is discussed in the next section. The functions $H_{0}, I_{1}, I_{3}$, $W_{2}, M_{2}, K_{2}$ and $(\Delta \ln \mu)_{2}$ are determined algebraically from equations (92), (96), (97), (100), (101), (104) and (93), respectively.

\subsection{Exterior solutions and boundary conditions}

The equations derived in the previous subsection apply to the interior of the star. Outside the star, in the vacuum, we have $\psi_{1}=0, m(r)=M_{*}, e^{2 \nu}=1-2 M_{*} / r$ and $\Delta \ln \mu=0$ and the resulting equations can be solved explicitly. The solutions for which the metric $g_{\mu \nu}$ is asymptotically flat are obtained as

$$
\begin{aligned}
M_{0} & =\Delta M_{*}, \\
H_{0} & =-\frac{\Delta M_{*}}{r-2 M_{*}}, \\
I_{1} & =I_{3}=0 \\
V_{1} & =\frac{2 \Delta J}{r}, \\
V_{3} & =\frac{\Delta V}{8 M_{*}^{3}} V_{f}\left(\frac{r}{2 M_{*}}\right), \\
W_{2} & =0 \\
H_{2} & =\frac{5 \Delta Q}{8 M_{*}^{3}} Q_{2}^{2}\left(\frac{r}{M_{*}}-1\right), \\
Y_{2} & =-\frac{5 \Delta Q}{8 M_{*}^{3}} \frac{2 M_{*}}{\left[r\left(r-2 M_{*}\right)\right]^{1 / 2}} Q_{2}^{1}\left(\frac{r}{M_{*}}-1\right), \\
M_{2} & =-\left(r-2 M_{*}\right) H_{2},
\end{aligned}
$$

where $\Delta M_{*}, \Delta J, \Delta V$ and $\Delta Q$ are constants, $V_{f}$ is the function defined by

$$
V_{f}(z)=\frac{7}{2}\left[\frac{1}{z}+5+30 z-210 z^{2}+180 z^{3}+60 z^{2}\left(3 z^{2}-5 z+2\right) \ln \left(1-\frac{1}{z}\right)\right],
$$

and $Q_{n}^{m}$ are the associated Legendre functions of the second kind,

$$
\begin{aligned}
& Q_{2}^{2}(z)=\frac{z\left(5-3 z^{2}\right)}{z^{2}-1}+\frac{3}{2}\left(z^{2}-1\right) \ln \frac{z+1}{z-1}, \\
& Q_{2}^{1}(z)=\frac{2-3 z^{2}}{\left(z^{2}-1\right)^{1 / 2}}+\frac{3}{2} z\left(z^{2}-1\right)^{1 / 2} \ln \frac{z+1}{z-1} .
\end{aligned}
$$


In the limit $z \rightarrow \infty$, we have $V_{f}(z) \rightarrow 1 / z^{3}, Q_{2}^{2}(z) \rightarrow 8 / 5 z^{3}$ and $Q_{2}^{1}(z) \rightarrow-2 / 5 z^{3}$. We may identify $\Delta M_{*}$ and $\Delta J$ with the mass shift and the angular momentum of the star, respectively, while $\Delta Q$ and $\Delta V$ with the mass quadrupole and current hexapole moments, respectively (Thorne 1980), apart from numerical factors.

Let us now explain our strategy to solve six unknown functions $M_{0},(\Delta \ln \mu)_{0}, V_{1}, V_{3}, Y_{2}$ and $H_{2}$. First, to obtain $M_{0}$ and $(\Delta \ln \mu)_{0}$, we need to specify what quantity is to be fixed when we add the perturbation. Although we may fix the central density (Hartle 1967; Hartle \& Thorne 1968; Chandrasekhar \& Miller 1974) or the total mass, here we adopt the baryon mass as the fixed parameter. From equation (1), the total baryon mass is given by (Hartle 1967)

$$
M_{B}=\int d^{3} x \sqrt{-g} \rho u^{0}
$$

Then, from equations (82) and (87), the perturbation of the total baryon mass up to $O\left(\Psi^{2}\right)$ is given by

$$
\Delta M_{B}=\int_{0}^{R_{*}} d r 4 \pi r^{2} e^{\lambda}\left[\frac{1}{r} e^{2 \lambda} \rho M_{0}+(\Delta \rho)_{0}\right] .
$$

This may be written in the differential form,

$$
\Delta M_{B}^{\prime}(r)=4 \pi r^{2} e^{\lambda}\left[\frac{1}{r} e^{2 \lambda} \rho M_{0}+\rho \mu \frac{d \rho}{d p}(\Delta \ln \mu)_{0}\right],
$$

where $\Delta M_{B}(r)$ is the baryon mass within radius $r$, and we have used the equality $(\Delta \rho)_{0}=\rho \mu(d \rho / d p)(\Delta \ln \mu)_{0}$ for an isentropic fluid. To fix the baryon mass, we solve this equation with the boundary conditions $\Delta M_{B}(0)=0$ and $\Delta M_{B}\left(R_{*}\right)=0$, together with equations (94) for $M_{0}$ and (95) for $(\Delta \ln \mu)_{0}$. Near the origin these functions have the behaviors,

$$
\begin{aligned}
M_{0} & \rightarrow \frac{1}{3} r^{3}\left[4 \pi \mu_{c}^{2} \rho_{c} \frac{d \rho_{c}}{d p_{c}}(\Delta \ln \mu)_{0 c}+2 \alpha_{1}^{2}\right], \\
(\Delta \ln \mu)_{0} & \rightarrow(\Delta \ln \mu)_{0 c}, \\
\Delta M_{B} & \rightarrow \frac{4 \pi}{3} r^{3} \mu_{c} \rho_{c} \frac{d \rho_{c}}{d p_{c}}(\Delta \ln \mu)_{0 c},
\end{aligned}
$$

where $\alpha_{1}$ is the constant introduced in equation (79), i.e., $\psi_{1} \rightarrow r^{2} \alpha_{1}$, and the subscript 'c' means the value at the center of the star. With these initial behaviors, equations (94), (95) and (121) can be integrated from the center to the stellar surface. We first determine the central value $(\Delta \ln \mu)_{0 c}$ so as to satisfy the boundary condition $\Delta M_{B}\left(R_{*}\right)=0$. Then the value of $M_{0}$ at $r=R_{*}$ gives the mass shift $\Delta M_{*}$ from equation (107), and the solution for $(\Delta \ln \mu)_{0}$, together with equations (92) and (108), determines the constant $D_{2}$.

Next, to obtain $V_{1}$, it is convenient to express it as the sum of a particular solution and a homogeneous solution

$$
V_{1}=V_{1}^{(P)}+c_{1} V_{1}^{(H)}
$$

where $c_{1}$ is a constant and the homogeneous solution $V_{1}^{(H)}$ satisfies equation (98) with the right-hand side being zero. Near the origin, we have

$$
V_{1}^{(P)} \rightarrow r^{2}, \quad V_{1}^{(H)} \rightarrow r^{2}
$$

Then, $V_{1}^{(P)}$ and $V_{1}^{(H)}$ can be obtained by integrating equation (98) from the center with the initial behavior (126) to the stellar surface. The constant $c_{1}$ in equation (125) and the angular momentum $\Delta J$ in equation (110) can be determined by requiring the continuities of the value $V_{1}$ and its derivative $V_{1}^{\prime}$ with the exterior solution in equation (110).

A similar method can be applied to equation (99) for $V_{3}$. In this case, the behavior near the origin is

$$
V_{3}^{(P)} \rightarrow r^{4}, \quad V_{3}^{(H)} \rightarrow r^{4}
$$

Then the solution determines the current hexapole moment $\Delta V$ from equation (111).

Finally, to obtain $Y_{2}$ and $H_{2}$, we again express them as the sum of a particular solution and a homogeneous solution

$$
Y_{2}=Y_{2}^{(P)}+c_{2} Y_{2}^{(H)}, \quad H_{2}=H_{2}^{(P)}+c_{2} H_{2}^{(H)},
$$

where $c_{2}$ is a constant and the homogeneous solutions $Y_{2}^{(H)}$ and $H_{2}^{(H)}$ satisfy equations (105) and (106), respectively, with the right-hand side being zero. Near the origin, they behave as

$$
\begin{aligned}
& Y_{2}^{(P)} \rightarrow \frac{r^{4}}{6}\left(\tilde{L}^{2} e^{-2 \nu_{c}} \alpha_{1}^{2}-8 \pi \rho_{c} e^{-\nu_{c}} D_{1} \alpha_{1}\right), \quad H_{2}^{(P)} \rightarrow \frac{8}{3} r^{2} \alpha_{1}^{2}, \\
& Y_{2}^{(H)} \rightarrow-\frac{2 \pi}{3} r^{4}\left(\rho_{c}+\rho_{c} \epsilon_{c}+3 p_{c}\right), \quad H_{2}^{(H)} \rightarrow r^{2} .
\end{aligned}
$$

These functions can be calculated by integrating equations (105) and (106) from the center with the initial behaviors (129) and (130) to the stellar surface. The constant $c_{2}$ in equation (128) and the mass quadrupole moment $\Delta Q$ in equations (113) and (114) can be determined by requiring the continuities of $Y_{2}$ and $H_{2}$ with the exterior solutions (113) and (114). 


\section{NUMERICAL RESULTS}

In this section we present the results of our numerical integration of the linearized GS equation (75) and the metric perturbation equations in $\S 4$. We adopt the fifth-order Runge-Kutta method with the adaptive stepsize control (Press et al. 1992) and use it iteratively when necessary. We adopt the polytropic equation of state

$$
p=K \rho^{1+1 / n}
$$

with the polytropic index $n=1$. We consider the non-rotating case $\Omega=0$ to concentrate on the magnetic effects.

\subsection{Magnetic field and fluid flow structure}

The GS equation (75) for $\psi_{1}$ is the basic equation to obtain the fluid flow and the electromagnetic field. The boundary condition (78) is satisfied for discrete eigenvalues $\tilde{L}$. The first few roots of the (dimensionless) eigenvalue $R_{*} \tilde{L}$ are given in Table 1 for several relativistic factors $M_{*} / R_{*}$. Although $\tilde{L}$ can take both signs, we assume $\tilde{L}>0$ in the following.

The components of the magnetic field $B^{\mu}$ are given by equations (83) - (86). Although the magnetic field is defined in the fluid frame in equation (5), the fluid 4-velocity $u^{\mu}$ there may be identified with the unit vector $n^{\mu}$ normal to the $t=$ const. hypersurface to the accuracy of $O(\Psi)$. Therefore, it is convenient to express the magnetic field in terms of the orthonormal tetrad components in the background metric (52). They are given by

$$
\begin{aligned}
B_{\hat{r}} & =-\frac{2}{r^{2}} \psi_{1} \cos \theta, \\
B_{\hat{\theta}} & =\frac{1}{r} e^{-\lambda} \psi_{1}^{\prime} \sin \theta, \\
B_{\hat{\varphi}} & =\frac{\tilde{L}}{r} e^{-\nu} \psi_{1} \sin \theta .
\end{aligned}
$$

In Figures 1] we show $B_{\hat{r}}(\theta=0), B_{\hat{\theta}}(\theta=\pi / 2)$ and $B_{\hat{\varphi}}(\theta=\pi / 2)$ normalized by $2 \alpha_{1}$ as a function of radius $r$ for the smallest two eigenvalues of $\tilde{L}$. Note that the magnetic field strength at the center of the star is given by $B_{c}^{2}=\left(2 \alpha_{1}\right)^{2}$. From these figures, we can see that the magnetic structure is more complicated for a larger eigenvalue $\tilde{L}$. To be precise, the number of nodes at which the $r$-derivative of the magnetic field vanishes increases as $\tilde{L}$ becomes large. This is also illustrated in Figures 2 in which the magnetic field lines projected on the meridional plane, i.e., the $\Psi=$ const. lines in the $(r, \theta)$-plane, are shown. In Figure 3. we show the projection of a truncated piece of a magnetic field line onto the equatorial plane. Only the part of it in the upper hemisphere $(\theta<\pi / 2)$ is shown. As we can see from the figure, the flux surface forms a torus and the magnetic field line winds around the torus.

The fluid flow is determined from equations (87) - (90). The orthonormal tetrad components of the fluid velocities in the background metric (52) are given by

$$
\begin{aligned}
& v_{\hat{r}}=\frac{2}{\tilde{C} \rho r^{2}} e^{-\nu}\left(\psi_{1}\right)^{2} \sin ^{2} \theta \cos \theta, \\
& v_{\hat{\theta}}=-\frac{1}{\tilde{C} \rho r} e^{-\lambda-\nu} \psi_{1} \psi_{1}^{\prime} \sin ^{3} \theta, \\
& v_{\hat{\varphi}}=\Omega e^{-\nu} r \sin \theta-\frac{\tilde{L}}{\tilde{C} \rho r} e^{-2 \nu}\left(\psi_{1}\right)^{2} \sin ^{3} \theta,
\end{aligned}
$$

where we set $\Omega=0$ in the present analysis. In Figures 4 we show $v_{\hat{r}}(\theta=\pi / 4), v_{\hat{\theta}}(\theta=\pi / 2)$ and $v_{\hat{\varphi}}(\theta=\pi / 2)$ as functions of radius $r$ for the smallest two eigenvalues of $\tilde{L}$, normalized by the dimensionless quantity,

$$
\mathcal{R}_{V}:=\frac{M_{*} R_{*}}{\tilde{C}} \frac{R_{*}^{4} \alpha_{1}^{2}}{M_{*}^{2}} .
$$

As in the case of the magnetic field, the velocity field is more complicated for a larger eigenvalue. In Figure 5 we plot the velocity field on the meridional plane $(\varphi=$ const. plane). The flow line resides on the constant flux surface.

In equation (138), the dimensionless quantity,

$$
\mathcal{R}_{M}:=\frac{R_{*}^{4} \alpha_{1}^{2}}{M_{*}^{2}}=\frac{3}{2} \frac{\left(B_{c}^{2} / 8 \pi\right)\left(4 \pi R_{*}^{3} / 3\right)}{M_{*}^{2} / R_{*}},
$$

describes the ratio of the magnetic energy to the gravitational energy, apart from a numerical factor. The quantity $\tilde{C}$ controls the magnitude of the meridional flow. As mentioned in $\S 3.2$ our perturbation method is valid as long as

$$
|\tilde{C}| \gg\left|R_{*}^{3} \alpha_{1}\right|=O(\Psi) .
$$

The limit $|\tilde{C}| \rightarrow \infty$ corresponds to the limit of zero meridional flow. 


\subsection{Mass shift and magnetic helicity}

By integrating the metric perturbation equations in $\S$ according to the prescription of $\S$ 4.2. we can calculate the change in the central density $(\Delta \rho)_{0 c}$, the mass shift $\Delta M_{*}$, the angular momentum $\Delta J$, the mass quadrupole moment $\Delta Q$ and the current hexapole moment $\Delta V$. In Table 2 we list the numerical results for several configurations. Each value is normalized appropriately as indicated in the first column. Here the dimensionless variable $\mathcal{R}_{V}$ defined in equation (138) measures the magnitude of the meridional flow velocity and $\mathcal{R}_{M}$ defined in equation (139) measures the ratio of the magnetic energy to the gravitational energy. We also introduce the dimensionless magnetic helicity $\mathcal{H}_{M}$ as defined in equation (145) below. The numbers given in Table 2 can be easily converted to physical units once we specify the mass $M_{*}$ and the dimensionless variables $\mathcal{R}_{V}$ (or $\tilde{C}$ ) and $\mathcal{R}_{M}$ (or $\alpha_{1}$ ).

The mass shift $\Delta M_{*} / M_{*} \mathcal{H}_{M}$ increases for larger eigenvalue $\tilde{L}$. This means that the configuration with the smallest eigenvalue $\tilde{L}=\tilde{L}_{1}$ is the lowest energy state for a fixed baryon mass $M_{B}$ and total magnetic helicity $\mathcal{H}_{M}$. The magnetic helicity is conserved in a perfect conducting plasma (Woltjer 1958), and it describes topological properties such as links, twists and kinks of magnetic field lines (Moffatt 1969; Berger \& Field 1984). When the topology of the field lines changes, the conductivity should be finite, which implies non-conservation of the magnetic helicity. However, it was conjectured that the total magnetic helicity is still approximately conserved on reconnection or relaxation time scales (Taylor 1974; Berger 1984). This hypothesis has been successful in laboratory plasmas (Taylor 1986). It is uncertain whether or not the total magnetic helicity is nearly conserved in the neutron star. In any case, we may speculate that the configuration with the smallest eigenvalue is preferred if the free energy could be released by the rearrangement of the magnetic field (see also Ioka 2001).

In the covariant language, the 4-current of magnetic helicity is defined (Carter 1978; Bekenstein 1987) by

$$
H^{\mu}=\frac{1}{2} \epsilon^{\mu \nu \alpha \beta} A_{\nu} F_{\alpha \beta}
$$

This is conserved in the ideal MHD,

$$
H^{\mu} ; \mu=0 \text {. }
$$

Thus the total magnetic helicity is defined by $\int d^{3} x \sqrt{-g} H^{0}$. Although the 4-current of magnetic helicity $H^{\mu}$ is not gauge-invariant locally, the total magnetic helicity is gauge-invariant for confined magnetic fields. From equations (14), (87) and (90) we have

$$
F_{12}=\tilde{L} e^{\lambda-\nu} \psi_{1} \sin \theta .
$$

Hence, using the gauge freedom to take $A_{2}=0$, we find

$$
A_{1}=\tilde{L} e^{\lambda-\nu} \psi_{1} \cos \theta \text {. }
$$

Therefore, we define the (dimensionless) total magnetic helicity by

$$
\mathcal{H}_{M}:=\frac{1}{M_{*}^{2}} \int d^{3} x \sqrt{-g} H^{0}=\frac{1}{M_{*}^{2}} \int_{0}^{R_{*}} d r \frac{16 \pi}{3} \tilde{L} e^{\lambda-\nu}\left(\psi_{1}\right)^{2} .
$$

\subsection{Spherical and quadrupolar deformations}

The magnetic stress distorts a star. The isobaric $\left(p=\right.$ const.) surface that lies at a radius $r_{0}$ in the background configuration is displaced in the perturbed configuration to a radius

where, from equation (55) and $(\Delta p)_{\ell}=\rho(\Delta \mu)_{\ell}$, we have

$$
r=r_{0}+(\Delta r)_{0}\left(r_{0}\right)+(\Delta r)_{2}\left(r_{0}\right) P_{2}(\cos \theta)
$$

$$
(\Delta r)_{\ell}=\frac{1}{\nu^{\prime}}(\Delta \ln \mu)_{\ell} \quad(\ell=0,2) .
$$

The spherical deformation is characterized by the monopole displacement $(\Delta r)_{0}$. However, to describe the quadrupolar deformation in a geometrically invariant manner, the coordinate radius $r$ is not quite appropriate. Instead of $r$, the circumferential radius $r_{*}^{2}=r^{2}\left[1+2 K_{2} P_{2}(\cos \theta)\right]$ is more appropriate (Hartle \& Thorne 1968). Then the isobaric surface is expressed as

$$
r_{*}\left(r_{0}, \theta\right)=r_{0}+(\Delta r)_{0}\left(r_{0}\right)+\left[(\Delta r)_{2}\left(r_{0}\right)+r_{0} K_{2}\left(r_{0}\right)\right] P_{2}(\cos \theta)
$$

We describe the quadrupolar deformation by the ellipticity defined by

$$
e_{*}(r)=\frac{r_{*}(r, \pi / 2)-r_{*}(r, 0)}{r}=-\frac{3}{2}\left[\frac{(\Delta \ln \mu)_{2}}{r \nu^{\prime}}+K_{2}\right] .
$$

In Table 2 we also list $(\Delta r)_{0}$ and $e_{*}$ at the stellar surface $r=R_{*}$ for various configurations. We can see that the stars are more deformed for larger eigenvalues of $\tilde{L}$, for the baryon mass and total magnetic helicity fixed. This is intuitively consistent with the results in the previous subsection that the energy (the mass shift) is higher for larger $\tilde{L}$ for constant baryon mass and total magnetic helicity. We also find that the configurations are prolate rather than oblate since $e_{*}<0$. This is in contrast with the case of stars with only poloidal fields which are oblate rather than prolate (Bocquet et al. 1995; Konno, Obata, \& Kojima 1999). This difference is due to the fact that the magnetic stress of a toroidal field tends to make a star prolate, working like a rubber belt tightening up the equator of the star (Ostriker \& Gunn 1969; Ioka 2001). Our model is the first example that demonstrates the validity of this picture in relativistic stars. 


\subsection{Frame dragging}

In general relativity, inertial frames are dragged not only by the fluid flow but also by the magnetic field. The dragging of inertial frames manifests itself in the off-diagonal components of the metric (82). In addition to the $t \varphi$ component $\left(V_{\ell}\right)$ which is familiar in the case of rotating stars and Kerr black holes, there also exist the $t r$-component $\left(I_{\ell}\right)$ and the $r \varphi$-component $\left(W_{\ell}\right)$ in the Regge-Wheeler gauge. These components originate from the meridional flow and the toroidal magnetic field, which are incorporated into the relativistic stellar model for the first time in this paper.

The 4-velocity of an observer whose world line is orthogonal to the $t=$ const. hypersurface $\Sigma_{t}$ is given by $n^{\mu}$ in equation (21). To the lowest order in $\Psi$, the coordinate angular velocity and the coordinate radial velocity of such observers are given by

$$
\begin{aligned}
& \frac{d \varphi}{d t}=\frac{n^{3}}{n^{0}}=N^{\varphi}=\frac{V_{1}}{r^{2}}+\frac{V_{3}}{r^{2}} \frac{3}{2}\left(5 \cos ^{2} \theta-1\right) \\
& \frac{d r}{d t}=\frac{n^{1}}{n^{0}}=N^{1}=-e^{-2 \lambda} I_{1} \cos \theta-e^{-2 \lambda} I_{3} \frac{1}{2}\left(5 \cos ^{3} \theta-3 \cos \theta\right),
\end{aligned}
$$

from equations (27), (29) and (82).

In Figures 6] we show the $t \varphi$-component of the metric perturbation that describes the coordinate angular velocity, for $M_{*} / R_{*}=0.2$ (relativistic case) and $M_{*} / R_{*}=0.01$ (Newtonian case). The left panel shows the dipole $(\ell=1$ ) component of the (normalized) coordinate angular velocity $\left(V_{1} / r^{2}\right) /\left(M_{*} \mathcal{R}_{V} / R_{*}^{2}\right)$ and the right panel shows the hexapole $(\ell=3)$ component $\left(V_{3} / r^{2}\right) /\left(M_{*} \mathcal{R}_{V} / R_{*}^{2}\right)$. From these figures, we can see that the normalized coordinate angular velocity does not depend on the relativistic factor $M_{*} / R_{*}$ so much. Thus, if the velocity of the meridional flow is fixed, i.e., $\mathcal{R}_{V}=$ const., the coordinate angular velocity is nearly proportional to $\left(M_{*} / R_{*}\right)^{2}$ for fixed mass. Note that the $t \varphi$-component $\left(V_{\ell}\right)$ is gauge-invariant for stationary perturbations (see equation (D3) in Zerilli 1970).

In Figures 7 we show the dipole and hexapole components of the (normalized) coordinate radial velocity in equation (151), $-e^{-2 \lambda} I_{1} /\left(M_{*} \mathcal{R}_{V} / R_{*}\right)$ and $-e^{-2 \lambda} I_{3} /\left(M_{*} \mathcal{R}_{V} / R_{*}\right)$, respectively. From these figures, and from the left panel of Figures 4 where we see that the fluid velocity $v$ is about $v \sim 0.1 \mathcal{R}_{V}$, as an order of magnitude estimate, the coordinate radial velocity is found to be $\sim\left(M_{*} / R_{*}\right) v$. It is interesting to note that the presence of the components $I_{1}$ and $I_{3}$ violates the reflection symmetry about the equatorial plane, i.e., $I_{1}$ and $I_{3}$ are of parity (-1) in equation (82). Note that the $t r$-component $\left(I_{\ell}\right)$ is gauge-specific by itself (see equation (D4) in Zerilli 1970). For example, we may choose a gauge in which $g_{01}=0$. However, for such a gauge we will have $g_{02} \neq 0$. What is significant is that we have a new frame dragging effect due to non-vanishing $N_{\Sigma}{ }^{\mu}$ because of the noncircularity of the spacetime, as pointed out at the end of $\S 2 \mathrm{C}$.

In analogy with the above arguments, let us consider the components of the unit spacelike 4-vector $m^{\mu}$ orthogonal to the $t=$ const. and $\varphi=$ const. hypersurface $\Sigma_{t \varphi}$ in equation (23). To the lowest order in $\Psi$, from equations (28), (29) and (82), we have

$$
\frac{d r}{d \varphi}=\frac{m^{1}}{m^{3}}=M_{\Sigma}^{1}=3 e^{-2 \lambda} W_{2} \sin ^{2} \theta \cos \theta .
$$

In Figure 8, we show the quadrupole component of the (normalized) coordinate derivative $e^{-2 \lambda} W_{2} /\left(M_{*}^{2} \mathcal{H}_{M} / R_{*}\right)$ for $M_{*} / R_{*}=0.2$ and $M_{*} / R_{*}=0.01$. We find from this figure that $d r / d \varphi \propto M_{*} / R_{*}$ for fixed mass and total magnetic helicity. This 'frame dragging' effect originates from the magnetic field rather than the fluid flow, since the right-hand side of equation (100) does not depend on $\tilde{C}$ (or $\mathcal{R}_{V}$ ). The component $W_{2}$ also violates the reflection symmetry about the equatorial plane, i.e., $W_{2}$ is of parity $(-1)$ in equation (82). Note, again, that the $r \varphi$-component $\left(W_{\ell}\right)$ is gauge-specific (see equation (D3) in Zerilli 1970). For example, we can take a gauge in which $g_{13}=0$. However, once again, an important point is that we have non-vanishing $M_{\Sigma}^{\mu}$ because of the noncircularity, which is a gauge-invariant statement.

\section{SUMMARY}

We have investigated stationary axisymmetric configurations of magnetized stars in the framework of general relativistic ideal MHD. A toroidal magnetic field and meridional flow in addition to a poloidal magnetic field are incorporated into the relativistic stellar model for the first time. We have treated the magnetic field and meridional flow as perturbations, but no other approximations were made. We have restricted ourselves to a star with a dipole magnetic field, slow rotation and polytropic equation of state, and considered magnetic field configurations confined in the interior of the star.

The solutions are found to be characterized by discrete eigenvalues that describe the radial profiles of the magnetic field and fluid flow. Namely, the magnetic field and velocity field configurations become more complicated for larger eigenvalues. We have found that the stellar shape is prolate rather than oblate because of the stress of the toroidal magnetic field. For fixed total baryonic mass and magnetic helicity, more spherical stars are found to have lower energy. We have also found two new types of frame dragging that differ from the one in rotating stars and Kerr black holes. These effects are due to the presence of the meridional flow and toroidal magnetic field. Interestingly, these effects violate the reflection symmetry about the equatorial plane. This means that the magnetic field or the meridional flow naturally selects the preferred direction about the axis of symmetry. This mechanism may be related to the neutron star kick or the jet formation. 
KI is grateful to F. Takahara and H. Nakano for useful discussions. This work was supported in part by the Monbukagaku-sho Grant-in-Aid for Scientific Research, Nos. 00660 (KI) and 14047214 (MS).

\section{REFERENCES}

Bekenstein, J. D. 1987, ApJ, 319, 207

Bekenstein, J. D., \& Oron, E. 1978, Phys. Rev. D, 18, 1809

Bekenstein, J. D., \& Oron, E. 1979, Phys. Rev. D, 19, 2827

Berger, M. A. 1984, Geophys. Astrophys. Fluid Dyn., 30, 79

Berger, M. A., \& Field, G. B. 1984, J. Fluid Mech., 147, 133

Bocquet, M., Bonazzola, S., Gourgoulhon, E., \& Novak, J. 1995, A\&A, 301, 757

Bonazzola, S., \& Gourgoulhon, E. 1996, A\&A, 312, 675

Bonazzola, S., Gourgoulhon, E., Salgado, M., \& Marck, J. A. 1993, A\&A, 278, 421

Cardall, C. Y., Prakash, M., \& Lattimer, J. M. 2001, ApJ, 554, 322

Carter, B. 1969, J. Math. Phys., 10, 70

Carter, B. 1973, in Black Holes, edited by DeWitt, C., \& DeWitt, B. S. (Gordon and Breach: New York)

Carter, B. 1978, in Active Galactic Nuclei, edited by Hazard, C., \& Mitton, S. (Cambridge: Cambridge University Press)

Chandrasekhar, S. 1933, MNRAS, 93, 390

Chandrasekhar, S., \& Miller, J. C. 1974, MNRAS, 167, 63

Cutler, C. 2002, Phys. Rev. D, 66, 084025

Duncan, R. C., \& Thompson, C. 1992, ApJ, 392, L9

Gourgoulhon, E., \& Bonazzola, S. 1993, Phys. Rev. D, 48, 2635

Hartle, J. B. 1967, ApJ, 150, 1005

Hartle, J. B., \& Thorne, K. S. 1968, ApJ, 153, 807

Ioka, K. 2001, MNRAS, 327, 639

Ioka, K., \& Sasaki, M. 2003, Phys. Rev. D, 67, 124026

Ioka, K., \& Taniguchi, K. 2000, ApJ, 537, 327

Kluźniak, W., \& Ruderman, M. 1998, ApJ, 505, L113

Konno, K., Obata, T., \& Kojima, Y. 1999, A\&A, 352, 211

Kouveliotou, C., et al. 1998, Nature, 393, 235

Lichnerowicz, A. 1967, Relativistic Hydrodynamics and Magnetohydrodynamics (Benjamin: New York)

Maeda, K., Sasaki, M., Nakamura, T., \& Miyama, S. 1980, Prog. Theor. Phys., 63, 719

Melatos, A. 1999, ApJ, 519, L77

Mereghetti, S., Chiarlone, L., Israel, G. L., \& Stella, L. 2002, astro-ph/0205122
Moffatt, H. K. 1969, J. Fluid Mech., 35, 117

Nakamura, T. 1998, Prog. Theor. Phys., 100, 921

Nakamura, T., Oohara, K., \& Kojima, Y. 1987, Prog. Theor. Phys. Suppl., 90, 1

Novikov, I. D., \& Thorne, K. S. 1973, in Black Holes, edited by

DeWitt, C., \& DeWitt, B. S. (Gordon and Breach: New York)

Oron, A. 2002, Phys. Rev. D, 66, 023006

Ostriker, J. P., \& Gunn, J. E. 1969, ApJ, 157, 1395

Papapetrou, A. 1966, Ann. Inst. H. Poincaré A, 4, 83

Press, W. H., Teukolsky, S. A., Vetterling, W. T., \& Flannery, B. P. 1992, Numerical Recipes in Fortran (2d ed.; Cambridge: Cambridge Univ. Press)

Punsly, B. 2001, Black Hole Gravitohydromagnetics (SpringerVerlag: Heidelberg)

Regge, T., \& Wheeler, J. A. 1957, Phys. Rev., 108, 1063

Rezzolla, L., Ahmedov, B. J., \& Miller, J. C. 2001a, Found. Phys. 31,1051

Rezzolla, L., Ahmedov, B. J., \& Miller, J. C. 2001b, MNRAS, 322, 723

Sasaki, M. 1984, in Problems of Collapse and Numerical Relativity, edited by Bancel, D., \& Signore, M. (Reidel: Dordrecht)

Taylor, J. B. 1974, Phys. Rev. Lett., 33, 1139

Taylor, J. B. 1986, Rev. Mod. Phys., 58, 741

Thompson, C. 2001, astro-ph/0110679

Thompson, C., \& Duncan, R. C. 1993, ApJ, 408, 194

Thorne, K. S. 1980, Rev. Mod. Phys., 52, 299

Usov, V. V. 1992, Nature, 357, 472

Wheeler, J. C., Höflich, I. Yi, P., \& Wang, L. 2000, ApJ, 537, 810

Woltjer, L. 1958, Proc. Nat. Acad. Sci., 44, 489

Zanotti, O., \& Rezzolla, L. 2002, MNRAS, 331, 376

Zerilli, F. J. 1970, Phys. Rev. D, 2, 2141 
TABle 1 . The First Few Roots of the (Dimensionless) eigenvalue $R_{*} \tilde{L}$ IN EQUATION [75] ARE SHOWN FOR SEVERAL RELATIVISTIC FACTORS $M_{*} / R_{*}$. Note that THE CONFIGURATION $M_{*} / R_{*}=0.24$ IS UNSTABLE AGAINST RADIAL PERTURBATIONS.

\begin{tabular}{ccccc}
\hline \hline & $M_{*} / R_{*}=0.24$ & $M_{*} / R_{*}=0.2$ & $M_{*} / R_{*}=0.1$ & $M_{*} / R_{*}=0.01$ \\
\hline$\left|R_{*} \tilde{L}_{1}\right|$ & 2.8704 & 3.9079 & 5.7924 & 7.2638 \\
$\left|R_{*} \tilde{L}_{2}\right|$ & 4.1284 & 5.6017 & 8.3151 & 10.475 \\
$\left|R_{*} \tilde{L}_{3}\right|$ & 5.3393 & 7.2568 & 10.795 & 13.615 \\
$\left|R_{*} \tilde{L}_{4}\right|$ & 6.5432 & 8.9031 & 13.258 & 16.729 \\
$\left|R_{*} \tilde{L}_{5}\right|$ & 7.7448 & 10.545 & 15.711 & 19.829 \\
\hline
\end{tabular}

TABle 2. PARAMETERS OF WEAKLY MAGNETIZED STARS IN GENERAL RELATIVITY ARE SHOWN FOR SEVERAL RELATIVISTIC FACTORS $M_{*} / R_{*}$ AND EIGENVALUES $\tilde{L}$ Given in Table 1 Shown are $(\Delta \rho)_{0 c}$ (Central Density Shift), $\Delta M_{*}$ (MASS SHIFT), $\Delta J$ (ANGULAR MOMENTUM), $\Delta Q$ (MASS QUADRUPOLE MOMENT), $\Delta V$ (CURRENT HEXAPOLE MOMENT),$(\Delta r)_{0}(\ell=0$ DEFORMATION OF THE STELLAR SURFACE), $e_{*}$ (ELLIPTICITY AT THE STELlaR SURFACE), AND $\mathcal{H}_{M}$ (DiMENSIONLESS MAGNETIC HELICITY). ThE DIMENSIONLESS QUANTITIES $\mathcal{R}_{V}$ AND $\mathcal{R}_{M}$ DEFINED IN EQUATIONS (138 AND (139), RESPECTIVELY, MEASURE THE MAGNITUdE OF FLUID FLOW AND MAGNETIC FIELD ENERGY. The INTEGER SUfFIXED TO EACH ENTRY DENOTES THE POWERS OF 10 BY WHICH THE ENTRY NUMBER MUST BE MULTIPLIED (E.G., $1.1_{-2}$ MEANS $1.1 \times 10^{-2}$ ).

\begin{tabular}{|c|c|c|c|c|c|c|c|c|c|}
\hline$M_{*} / R_{*}$ & $\tilde{L}$ & $\frac{(\Delta \rho)_{0 c}}{\rho_{c} \mathcal{H}_{M}}$ & $\frac{\Delta M_{*}}{M_{*} \mathcal{H}_{M}}$ & $\frac{\Delta J}{M_{*} R_{*} \mathcal{R}_{V}}$ & $\frac{\Delta Q}{M_{*} R_{*}^{2} \mathcal{H}_{M}}$ & $\frac{\Delta V}{M_{*} R_{*}^{3} \mathcal{R}_{V}}$ & $\frac{(\Delta r)_{0}}{R_{*} \mathcal{H}_{M}}$ & $\frac{e_{*}}{\mathcal{H}_{M}}$ & $\frac{\mathcal{H}_{M}}{\mathcal{R}_{M}}$ \\
\hline $\begin{array}{c}0.24 \\
\left(\rho_{c} M_{*}^{2}=1.3304_{-2}\right) \\
\left(p_{c} / \rho_{c}=5.4344_{-1}\right) \\
\left(\epsilon_{c}=n p_{c} / \rho_{c}\right)\end{array}$ & $\begin{array}{l}\tilde{L}_{1} \\
\tilde{L}_{2} \\
\tilde{L}_{3} \\
\tilde{L}_{4} \\
\tilde{L}_{5}\end{array}$ & $\begin{array}{l}-1.7201_{-1} \\
-1.3834_{-1} \\
-1.3433_{-1} \\
-1.2457_{-1} \\
-1.2618_{-1}\end{array}$ & $\begin{array}{l}2.2123_{-2} \\
2.8090_{-2} \\
3.3088_{-2} \\
3.8033_{-2} \\
4.3057_{-2}\end{array}$ & $\begin{array}{l}-1.9030_{-2} \\
-3.7484_{-2} \\
-1.3583_{-2} \\
-1.7557_{-2} \\
-9.0243_{-3}\end{array}$ & $\begin{array}{l}-2.4813_{-3} \\
-7.5644_{-3} \\
-1.3770_{-2} \\
-2.0184_{-2} \\
-2.6556_{-2}\end{array}$ & $\begin{array}{l}1.6221_{-4} \\
5.0828_{-4} \\
2.3390_{-4} \\
3.3273_{-4} \\
1.7827_{-4}\end{array}$ & $\begin{array}{l}7.8089_{-2} \\
1.2146_{-1} \\
1.6951_{-1} \\
2.1765_{-1} \\
2.6526_{-1}\end{array}$ & $\begin{array}{l}-6.8901_{-3} \\
-2.1005_{-2} \\
-3.8237_{-2} \\
-5.6046_{-2} \\
-7.3741_{-2}\end{array}$ & $\begin{array}{l}1.8450_{-1} \\
2.2614_{-1} \\
7.4362_{-2} \\
9.2887_{-2} \\
4.7016_{-2}\end{array}$ \\
\hline $\begin{array}{c}0.2 \\
\left(\rho_{c} M_{*}^{2}=6.7418_{-3}\right) \\
\left(p_{c} / \rho_{c}=2.5582_{-1}\right) \\
\left(\epsilon_{c}=n p_{c} / \rho_{c}\right)\end{array}$ & $\begin{array}{l}\tilde{L}_{1} \\
\tilde{L}_{2} \\
\tilde{L}_{3} \\
\tilde{L}_{4} \\
\tilde{L}_{5}\end{array}$ & $\begin{array}{l}2.5443_{-1} \\
4.0620-1 \\
5.3048_{-1} \\
6.7339_{-1} \\
7.9653_{-1} \\
\end{array}$ & $\begin{array}{l}2.4450_{-2} \\
3.0662_{-2} \\
3.6320_{-2} \\
4.2004_{-2} \\
4.7794_{-2} \\
\end{array}$ & $\begin{array}{l}-2.5850_{-2} \\
-7.0990_{-2} \\
-2.0397_{-2} \\
-3.0716_{-2} \\
-1.4039_{-2} \\
\end{array}$ & $\begin{array}{l}-6.1618_{-3} \\
-1.8749_{-2} \\
-3.2930_{-2} \\
-4.7192_{-2} \\
-6.1198_{-2} \\
\end{array}$ & $\begin{array}{l}3.6056_{-4} \\
1.4779_{-3} \\
5.1754_{-4} \\
8.3493_{-4} \\
3.9211_{-4} \\
\end{array}$ & $\begin{array}{r}-2.5991_{-2} \\
9.0360_{-3} \\
4.1634_{-2} \\
7.3899_{-2} \\
1.0519_{-1} \\
\end{array}$ & $\begin{array}{l}-1.5050_{-2} \\
-4.5796_{-2} \\
-8.0431_{-2} \\
-1.1527_{-1} \\
-1.4948_{-1} \\
\end{array}$ & $\begin{array}{l}2.5561_{-1} \\
4.3463_{-1} \\
1.1531_{-1} \\
1.6900_{-1} \\
7.6323_{-2}\end{array}$ \\
\hline $\begin{array}{c}0.1 \\
\left(\rho_{c} M_{*}^{2}=7.9326_{-4}\right) \\
\left(p_{c} / \rho_{c}=7.0273_{-2}\right) \\
\left(\epsilon_{c}=n p_{c} / \rho_{c}\right)\end{array}$ & $\begin{array}{l}\tilde{L}_{1} \\
\tilde{L}_{2} \\
\tilde{L}_{3} \\
\tilde{L}_{4} \\
\tilde{L}_{5} \\
\end{array}$ & $\begin{array}{r}-4.2056_{-2} \\
1.1871_{-1} \\
1.8560_{-1} \\
3.0621_{-1} \\
3.8261_{-1} \\
\end{array}$ & $\begin{array}{l}1.7802_{-2} \\
2.2276_{-2} \\
2.6519_{-2} \\
3.0791_{-2} \\
3.5149_{-2} \\
\end{array}$ & $\begin{array}{l}-2.5913_{-2} \\
-8.7908_{-2} \\
-2.2024_{-2} \\
-3.6953_{-2} \\
-1.5645_{-2} \\
\end{array}$ & $\begin{array}{l}-1.9571_{-2} \\
-6.0171_{-2} \\
-1.0366_{-1} \\
-1.4668_{-1} \\
-1.8861_{-1} \\
\end{array}$ & $\begin{array}{l}6.8215_{-4} \\
3.2927_{-3} \\
9.7473_{-4} \\
1.7212_{-3} \\
7.4237_{-4} \\
\end{array}$ & $\begin{array}{l}9.0505_{-2} \\
1.8445_{-1} \\
2.7967_{-1} \\
3.7327_{-1} \\
4.6484_{-1} \\
\end{array}$ & $\begin{array}{l}-3.6533_{-2} \\
-1.1232_{-1} \\
-1.9350_{-1} \\
-2.7381_{-1} \\
-3.5208_{-1} \\
\end{array}$ & $\begin{array}{l}2.7778_{-1} \\
5.8027_{-1} \\
1.3491_{-1} \\
2.2085_{-1} \\
9.2516_{-2} \\
\end{array}$ \\
\hline $\begin{array}{c}0.01 \\
\left(\rho_{c} M_{*}^{2}=7.8563_{-7}\right) \\
\left(p_{c} / \rho_{c}=5.1447_{-3}\right) \\
\left(\epsilon_{c}=n p_{c} / \rho_{c}\right)\end{array}$ & $\begin{array}{l}\tilde{L}_{1} \\
\tilde{L}_{2} \\
\tilde{L}_{3} \\
\tilde{L}_{4} \\
\tilde{L}_{5}\end{array}$ & $\begin{array}{r}-1.0732_{-1} \\
1.1140_{-1} \\
1.8112_{-1} \\
3.4219_{-1} \\
4.3170_{-1}\end{array}$ & $\begin{array}{l}2.2215_{-3} \\
2.7927_{-3} \\
3.3291_{-3} \\
3.8697_{-3} \\
4.4214_{-3}\end{array}$ & $\begin{array}{l}-2.3000_{-2} \\
-7.9968_{-2} \\
-2.0174_{-2} \\
-3.4439_{-2} \\
-1.4475_{-2}\end{array}$ & $\begin{array}{l}-3.9173_{-2} \\
-1.2203_{-1} \\
-2.0944_{-1} \\
-2.9532_{-1} \\
-3.7878_{-1}\end{array}$ & $\begin{array}{l}8.9803_{-4} \\
4.3637_{-3} \\
1.2846_{-3} \\
2.2933_{-3} \\
9.7915_{-4}\end{array}$ & $\begin{array}{l}1.3237_{-1} \\
2.7151_{-1} \\
4.1207_{-1} \\
5.4972_{-1} \\
6.8415_{-1}\end{array}$ & $\begin{array}{l}-5.9957_{-2} \\
-1.8677_{-1} \\
-3.2056_{-1} \\
-4.5201_{-1} \\
-5.7975_{-1}\end{array}$ & $\begin{array}{l}2.6217_{-1} \\
5.5877_{-1} \\
1.3056_{-1} \\
2.1748_{-1} \\
9.0471_{-2}\end{array}$ \\
\hline
\end{tabular}



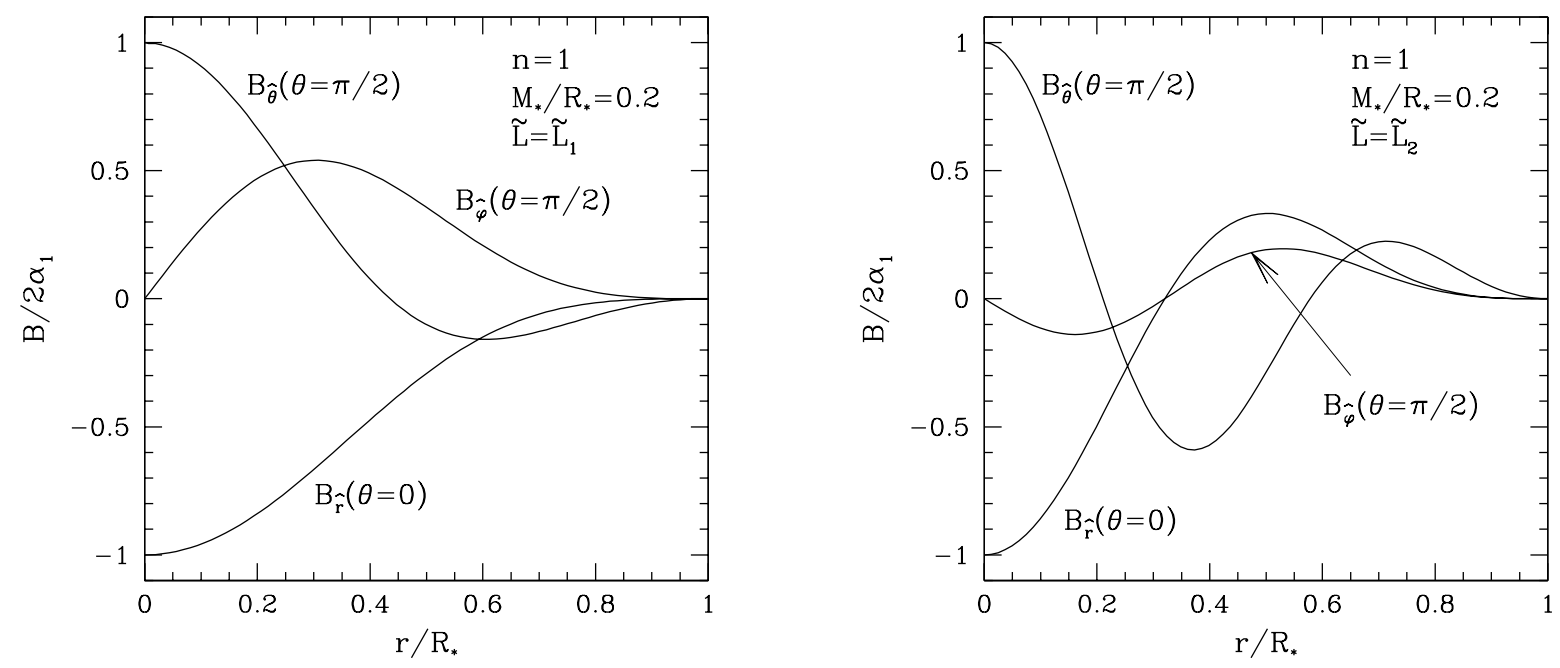

FIG. 1. - The components $B_{\hat{r}}(\theta=0), B_{\hat{\theta}}(\theta=\pi / 2)$ and $B_{\hat{\varphi}}(\theta=\pi / 2)$ of the magnetic field in the orthonormal basis in equations 132 - 134 normalized by the central magnetic field $2 \alpha_{1}$ are shown as functions of radius $r / R_{*}$. We adopt the polytropic index $n=1$, the relativistic factor $M_{*} / R_{*}=0.2$. The left panel shows the case of the eigenvalue $\tilde{L}=\tilde{L}_{1}$ and the right panel the case of $\tilde{L}=\tilde{L}_{2}$ given in Table 1
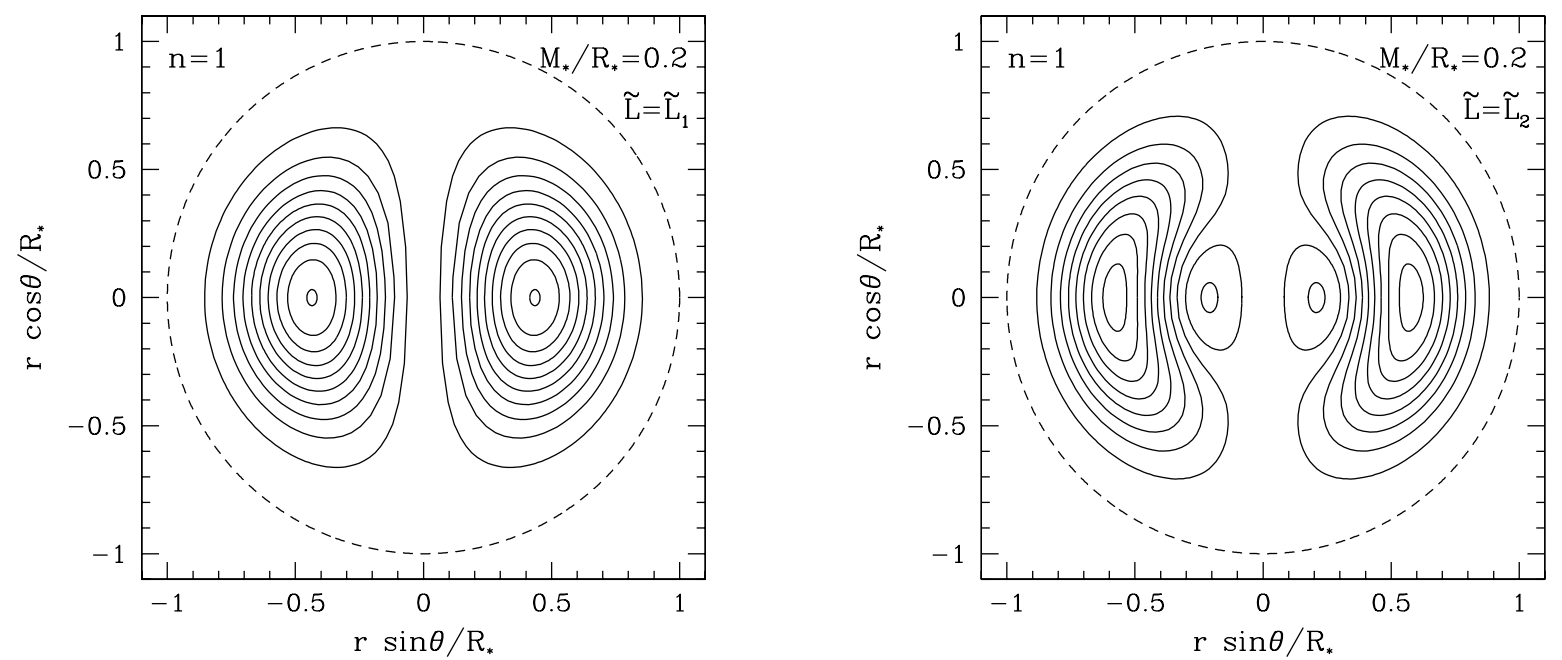

FIG. 2. - The magnetic field lines projected on the meridional plane, i.e., the $\Psi=$ const. lines in the $(r, \theta)$-plane. The dashed line is the stellar surface. We adopt the polytropic index $n=1$, the relativistic factor $M_{*} / R_{*}=0.2$. The left panel shows the case of the eigenvalue $\tilde{L}=\tilde{L}_{1}$ and the right panel the case of $\tilde{L}=\tilde{L}_{2}$ given in Table 1 


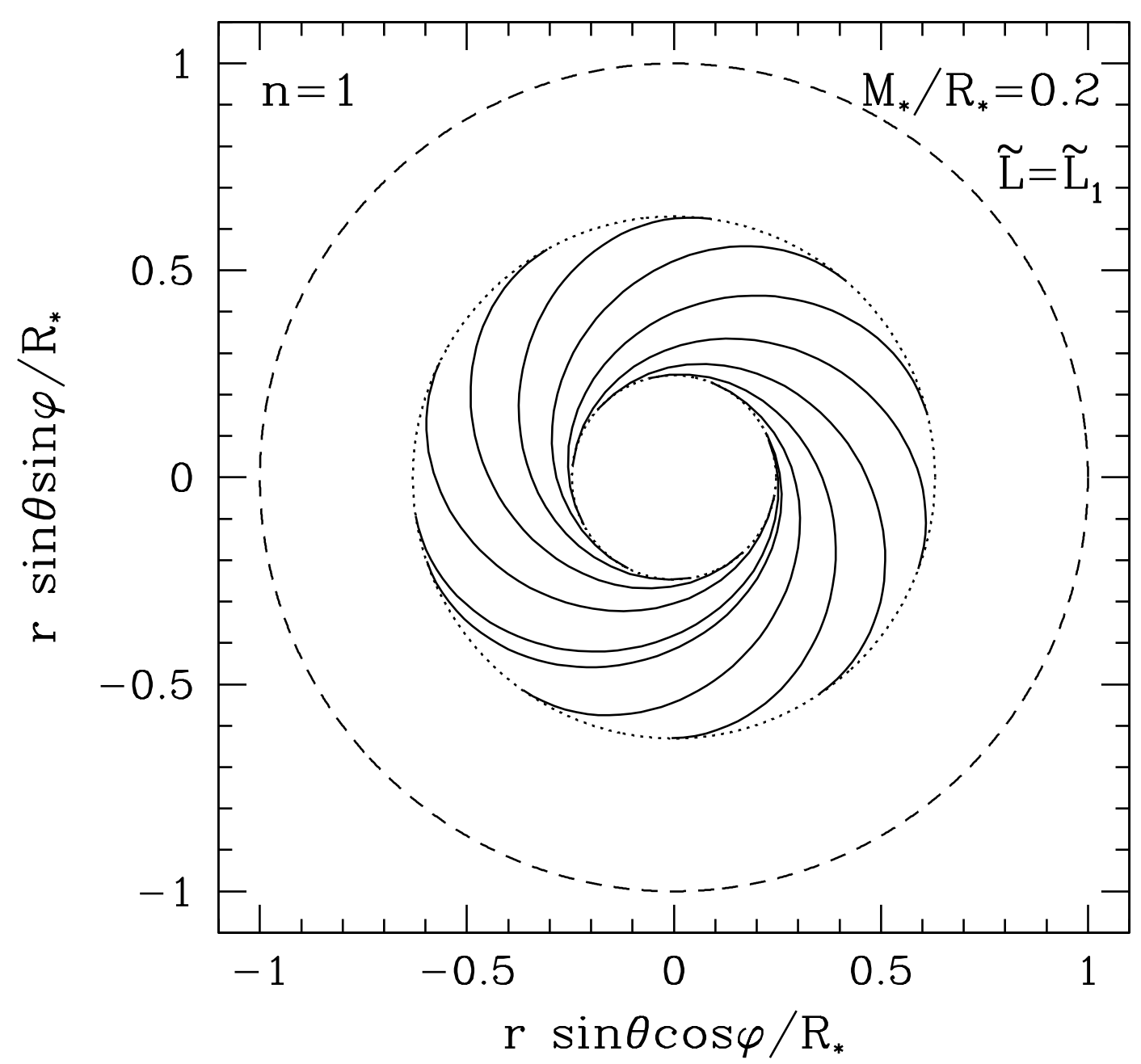

FIG. 3.- A truncated piece of the magnetic field line on a certain flux surface $(\Psi=$ const. surface) projected onto the equatorial plane $(\theta=\pi / 2$ plane). Only the part of it in the upper hemisphere $(\theta<\pi / 2)$ is shown. The dashed line is the stellar surface. The dotted lines are the cross section of the flux surface with the equatorial plane. We adopt the polytropic index $n=1$, the relativistic factor $M_{*} / R_{*}=0.2$, and the eigenvalue $\tilde{L}=\tilde{L}_{1}$ in Table 1 

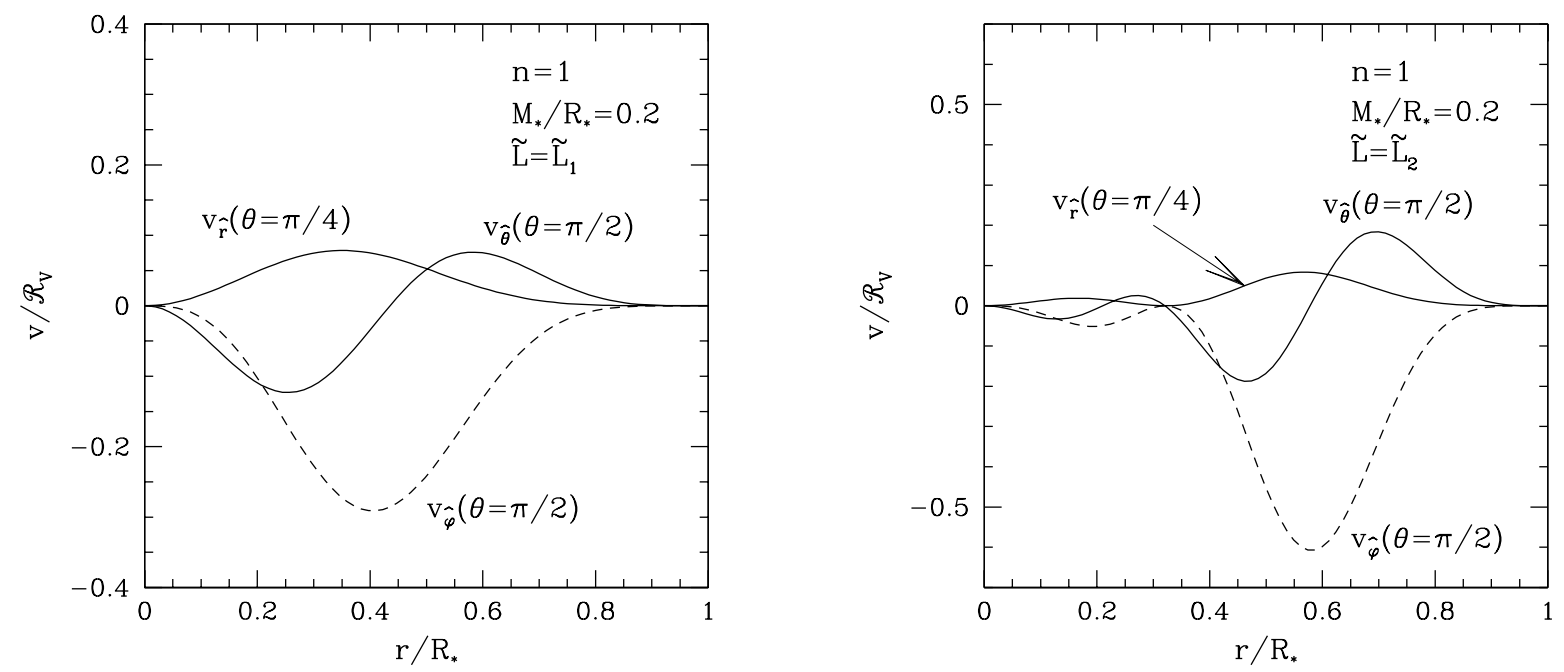

FIG. 4.- The components $v_{\hat{r}}(\theta=\pi / 4), v_{\hat{\theta}}(\theta=\pi / 2)$ and $v_{\hat{\varphi}}(\theta=\pi / 2)$ of the fluid velocity in the orthonormal basis in equations 135 137, normalized by the dimensionless variable $\mathcal{R}_{V}$ in equation 138, as functions of radius $r / R_{*}$. We adopt the polytropic index $n=1$, the relativistic factor $M_{*} / R_{*}=0.2$. The left panel shows the case of the eigenvalue $\tilde{L}=\tilde{L}_{1}$ and the right panel the case of $\tilde{L}=\tilde{L}_{2}$ given in Table 1 


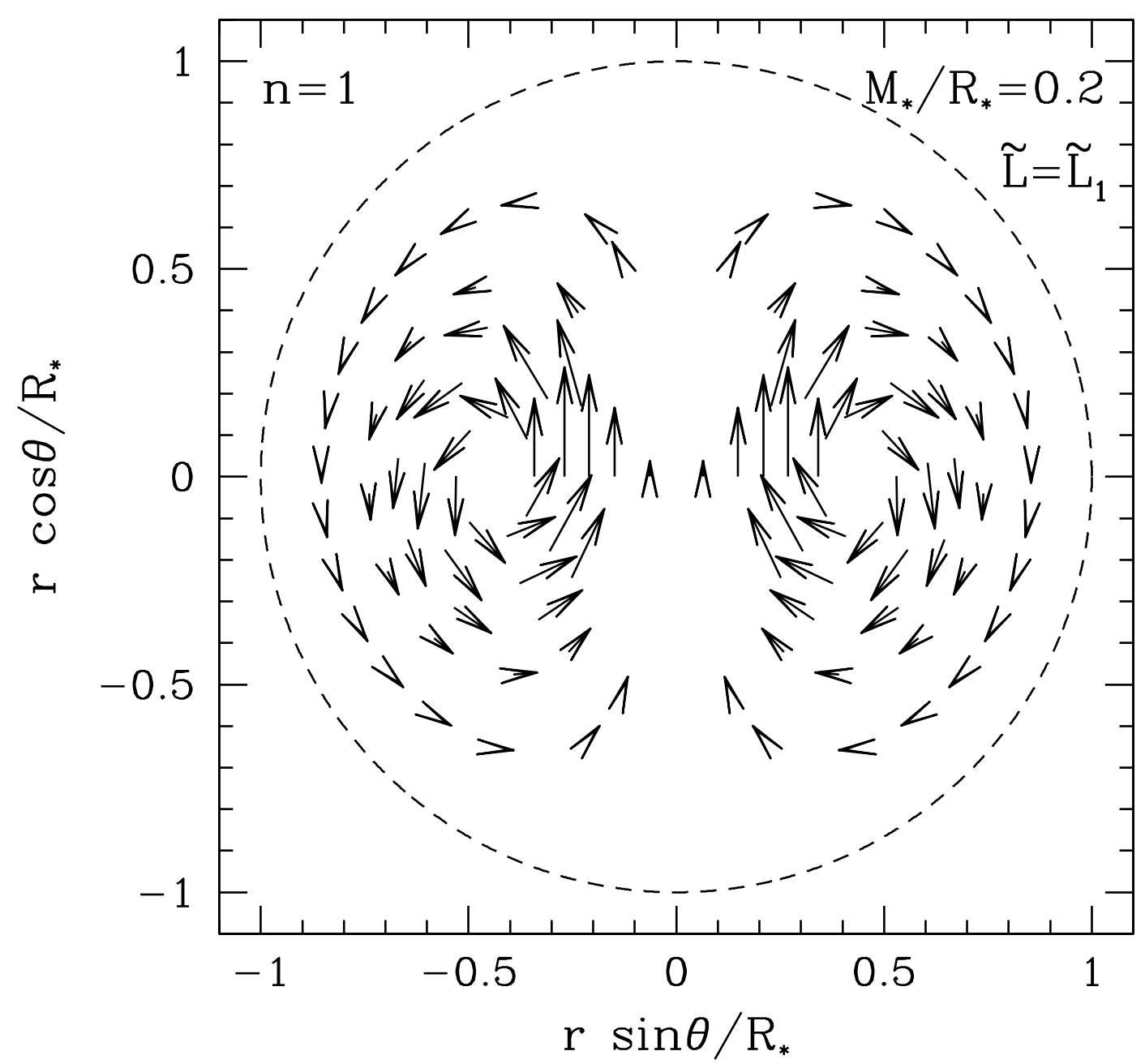

FIG. 5.- The velocity field in the meridional plane ( $\varphi=$ const. plane). The lengths of the vectors are proportional to the fluid velocity in the orthonormal basis in equations (135 - 137). The dashed line is the stellar surface. We adopt the polytropic index $n=1$, the relativistic factor $M_{*} / R_{*}=0.2$, and the eigenvalue $\tilde{L}=\tilde{L}_{1}$ in Table 

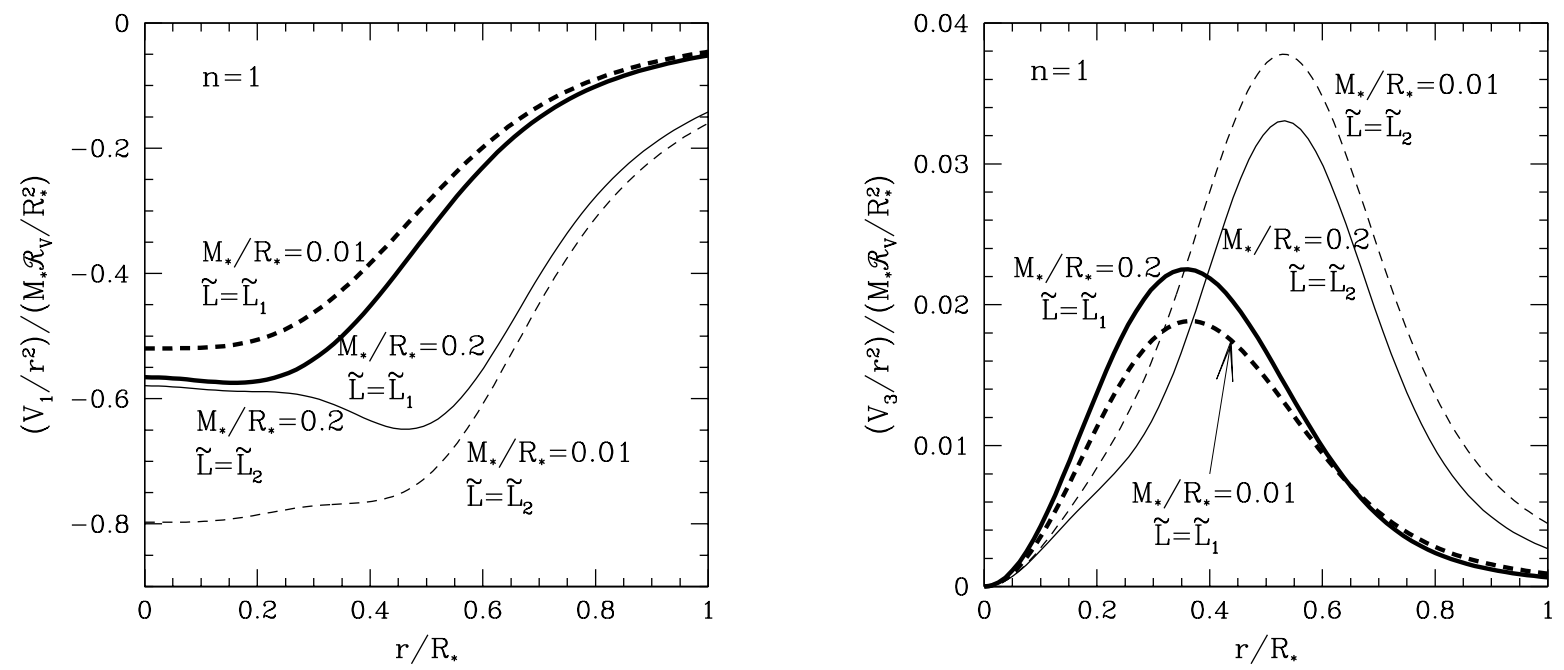

FIG. 6. - The $t \varphi$-component of the dipole $(\ell=1)$ metric perturbation (left panel) and that of the hexapole $(\ell=3)$ metric perturbation (right panel) as functions of radius, namely, $V_{1} / r^{2}$ normalized by $M_{*} \mathcal{R}_{V} / R_{*}^{2}$ and $V_{3} / r^{2}$ normalized by $M_{*} \mathcal{R}_{V} / R_{*}^{2}$, respectively. The solid and dashed lines are for $M_{*} / R_{*}=0.2$ (relativistic) and $M_{*} / R_{*}=0.01$ (Newtonian), respectively, while the bold and thin lines for $\tilde{L}=\tilde{L}_{1}$ and $\tilde{L}=\tilde{L}_{2}$ in Table 1 respectively. We adopt the polytropic index $n=1$. These metric components describe the standard frame dragging due to the angular momentum.
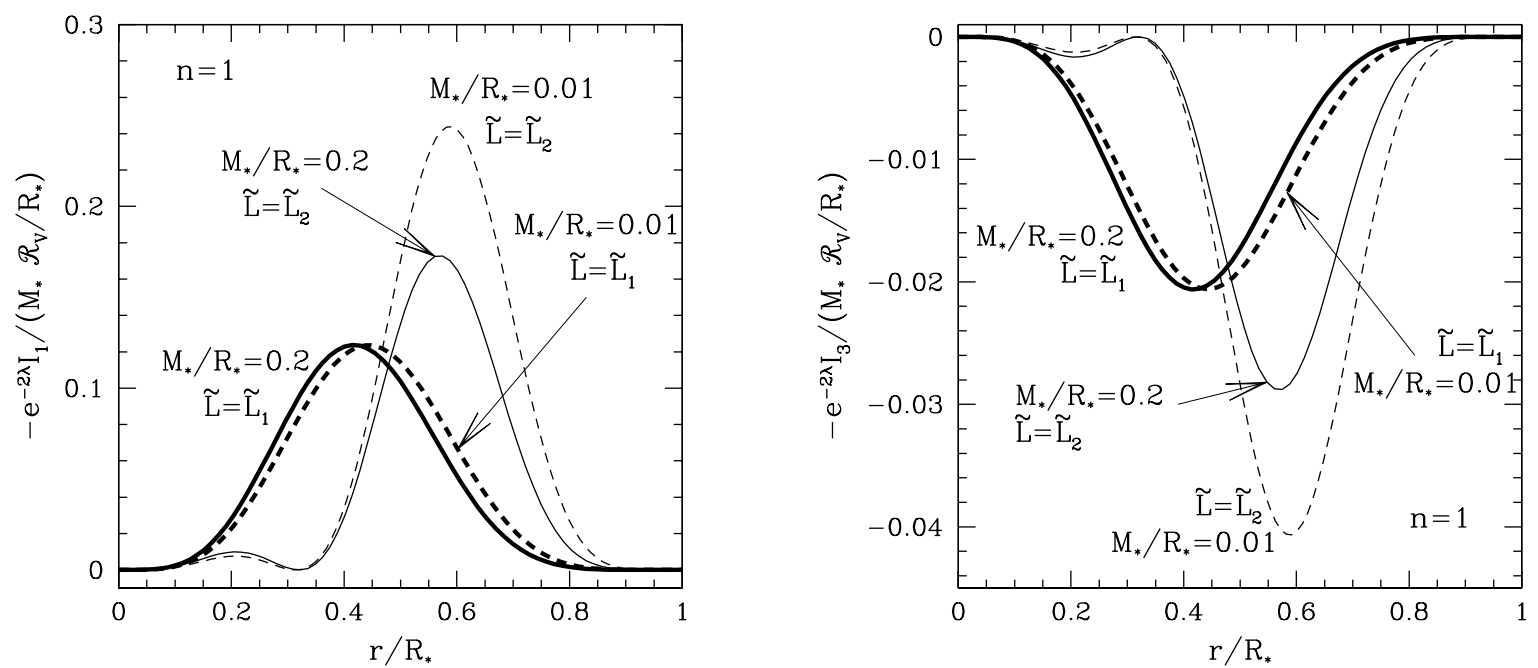

Fig. 7. - The tr-component of the dipole $(\ell=1)$ metric perturbation (left panel) and that of the hexapole $(\ell=3)$ metric perturbation (right panel) as functions of radius, namely, $-e^{-2 \lambda} I_{1}$ normalized by $M_{*} \mathcal{R}_{V} / R_{*}$ and $-e^{-2 \lambda} I_{3}$ normalized by $M_{*} \mathcal{R}_{V} / R_{*}$, respectively. The solid and dashed lines are for $M_{*} / R_{*}=0.2$ (relativistic) and $M_{*} / R_{*}=0.01$ (Newtonian), respectively, while the bold and thin lines for $\tilde{L}=\tilde{L}_{1}$ and $\tilde{L}=\tilde{L}_{2}$ in Table 1 respectively. We adopt the polytropic index $n=1$. These metric components describe a new type of frame dragging due to meridional flow. 


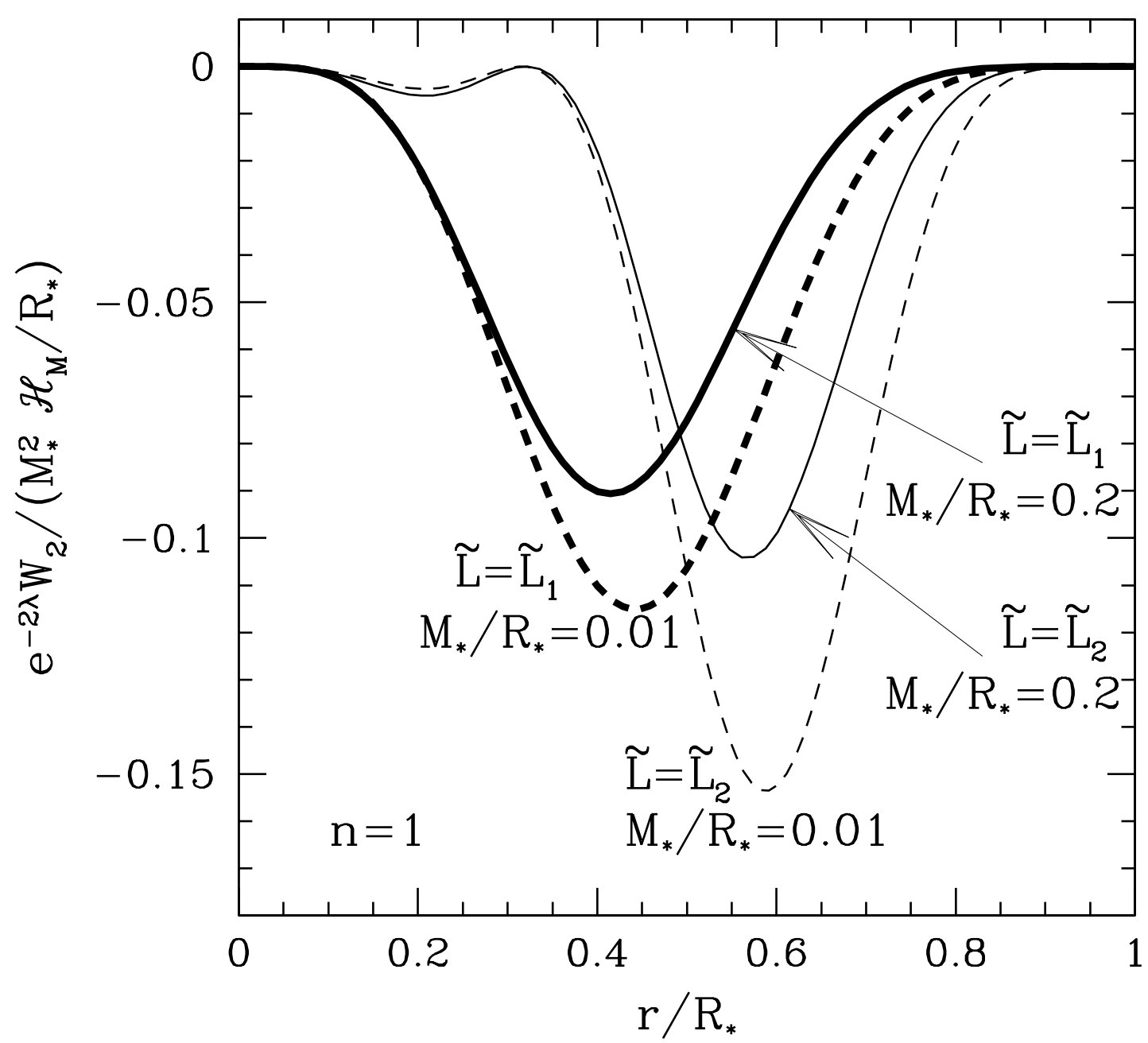

FIG. 8. - The $r \varphi$-component of the quadrupole $(\ell=2)$ metric perturbation as a function of radius, namely, $e^{-2 \lambda} W_{2}$ normalized by $M_{*}^{2} \mathcal{H}_{M} / R_{*}$. The solid and dashed lines are for $M_{*} / R_{*}=0.2$ (relativistic) and $M_{*} / R_{*}=0.01$ (Newtonian), respectively, while the bold and thin lines for $\tilde{L}=\tilde{L}_{1}$ and $\tilde{L}=\tilde{L}_{2}$ in Table 1 respectively. We adopt the polytropic index $n=1$. This metric component describes a new type of frame dragging due to a toroidal magnetic field. 